\title{
Zum Mechanismus der Carbodesilylierung 4- bzw. 5-substituierter 2-(Trimethylsilyl)pyridine mit Benzaldehyd ${ }^{[1]}$
}

\author{
Franz Effenberger*, Andreas Krebs ${ }^{\mid 2 a l}$ und Peter Willrett ${ }^{\mid 2 b]}$ \\ Institut für Organische Chemie der Universität Stuttgart, \\ Pfaffenwaldring 55, W-7000 Stuttgart 80
}

Eingegangen am 28. September 1991

Key Words: Pyridines, substituted 2-(trimethylsilyl)-, synthesis of, carbodesilylation of / Carbodesilylation / Rate constants

On the Mechanism of the Carbodesilylation of 4- or 5-Substituted 2-(Trimethylsilyl)pyridines

An "Ylide mechanism" is proposed for the carbodesilylation of 2-(trimethylsilyl)pyridines with benzaldehyde. In contrast, 3and 4-(trimethylsilyl)pyridines, react only in the presence of a base catalyst via pyridyl anions with electrophiles. The rates of the uncatalyzed carbodesilylation reactions of 4-substituted 2-(trimethylsilyl)pyridines 2 with benzaldehyde correlate very well with the resonance parameters of the substituents $\sigma_{R}^{0}$, whereas the rates of 5-substituted 2-(trimethylsilyl)pyridines 7 correlate with the inductive substituent parameters $\sigma_{I}$ in the Taft equation. This is to our knowledge the first direct determination of the resonance parameters $\sigma_{\mathrm{R}}^{o}$.
Die Carbodesilylierung Trimethylsilyl-substituierter Aromaten bzw. Heteroaromaten mit Aldehyden, Ketonen, Carbonsäuren oder Kohlensäurederivaten stellt einen bequemen Zugang zur Einführung von Kohlenstoffsubstituenten in diese Verbindungen dar. Bei elektronenreichen Aromaten läuft die Carbodesilylierung als elektrophile Substitution über Wheland-Zwischenstufen $\mathrm{ab}^{[3]}$, während bei Akzeptorsubstituierten Verbindungen Aryl-Anionen entscheidende Zwischenstufen sind ${ }^{[4]}$. Carbodesilylierungen über ArylAnionen als Zwischenstufen sind Base-katalysierte Reaktionen $^{[4]}$. Bei Carbodesilylierungen Trimethylsilyl-substituierter Heteroaromaten mit Azin-Stickstoff, die mit Akzeptor-substituierten Benzolen vergleichbar sind, ist nur bei der Substitution von Trimethylsilylgruppen, die nicht zum Azin$\mathrm{N}$ benachbart sind, eine Katalyse mit Basen erforderlich ${ }^{[5]}$.

Heterocyclen, bei denen die zu substituierende Trimethylsilylgruppe in Nachbarstellung zum Azin-N steht, reagieren auch ohne Zusatz von Basen ${ }^{[4 \mathrm{~b}, \mathrm{c}]}$ unter relativ milden Bedingungen mit Elektrophilen ${ }^{[6,7]}$. Der Grund hierfür wurde von Jutzi et al ${ }^{[8]}$ in einer primären Reaktion des freien Elektronenpaares am Ringstickstoff mit dem Elektrophil unter Ausbildung eines Aziniumsalzes gesehen, das dann unter Ylidbildung besonders leicht eine $\mathrm{Si}-\mathrm{C}$-Bindungsspaltung eingeht. Eigene Untersuchungen zum Mechanismus dieser Reaktion an 2-Trimethylsilyl-substituierten Pyridinen ${ }^{[2 a]}$ und an (Trimethylsilyl)estern von Heterocyclen ${ }^{[9]}$ sprechen für den vorgeschlagenen Mechanismus ${ }^{[8]}$.

In der vorliegenden Publikation berichten wir über die Beeinflussung der Geschwindigkeit der Carbodesilylierung von 2-(Trimethylsilyl)pyridinen mit Benzaldehyd durch unterschiedliche Substituenten in 4- bzw. 5-Position. Durch Wahl dieser Substituenten kann die Basizität der Pyridine einerseits und die Stabilität von Pyridyl-Anionen andererseits in definierter Weise verändert werden. Aus den Unterschieden der Reaktionsgeschwindigkeiten der substituierten 2-(Trimethylsilyl)pyridine sollten dann genauere Aussagen

über den Reaktionsmechanismus dieser Carbodesilylierungen bezüglich der Pyridinium- bzw. Ylidbildung möglich sein.

\section{Umsetzungen von 2-, 3- bzw. 4-(Trimethylsilyl)pyridin} (2a, 5a bzw. 5b) mit Benzaldehyd

Die Trimethylsilyl (TMS)-substituierten Pyridine 2a, 5a und $5 \mathbf{b}$ wurden aus den Halogenverbindungen $1 \mathbf{a}, \mathbf{4} \mathbf{a}$ und 4b mit Magnesium und Chlortrimethylsilan in Hexamethylphosphorsäuretriamid (HMPT) über eine In-situ-Grignard-Reaktion ${ }^{[10]}$ hergestellt.

\section{Schema 1}<smiles>Clc1ccccn1</smiles>

10
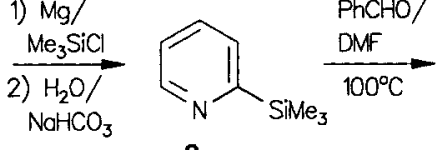

2a
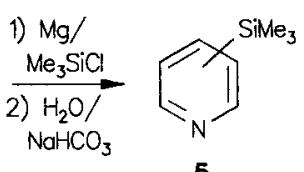

5
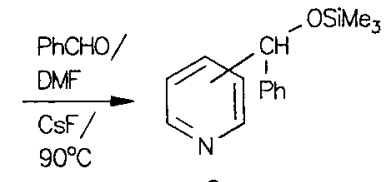

4

\begin{tabular}{c|ccc} 
& $\mathbf{4}$ & $\mathbf{5}$ & 6 \\
\hline a & $3-\mathrm{Br}$ & $3-\mathrm{SiMe}_{3}$ & $3-\mathrm{CH}\left(\mathrm{OSiMe}_{3}\right) \mathrm{Ph}$ \\
b & $4-\mathrm{Br}$ & $4-\mathrm{SiMe}_{3}$ & $4-\mathrm{CH}\left(\mathrm{OSiMe}_{3}\right) \mathrm{Ph}$
\end{tabular}

2a konnte entsprechend den Literaturangaben ${ }^{[6]}$ beim Erhitzen mit Benzaldehyd in Dimethylformamid (DMF) auf $100{ }^{\circ} \mathrm{C}$ mit $89 \%$ Ausbeute in das Carbodesilylierungspro- 
dukt 3a übergeführt werden. Unter vergleichbaren Bedingungen reagieren 3- bzw. 4-TMS-Pyridin $(\mathbf{5 a}, \mathbf{b})$ auch nach langen Reaktionszeiten nicht mit Benzaldehyd. Bei Zugabe von Caesiumfluorid als basischem Katalysator erfolgt dagegen bei beiden Verbindungen Carbodesilylierung zu 6a bzw. 6b (Schema 1).

Während bei $5 \mathbf{b}$ die Reaktion bei $90^{\circ} \mathrm{C}$ schon nach zwei Stunden beendet ist und in 74proz. Ausbeute $6 \mathrm{~b}$ ergibt, erfordert die Umsetzung von $\mathbf{5 a}$ eine Reaktionszeit von 34 Stunden. Neben den Silylethern 6 werden bei diesen Reaktionen z.T. auch Folgeprodukte dieser Verbindungen isoliert. Aus 5 a werden so neben $49 \%$ 6a noch $10 \% 3$-Benzoylpyridin gefunden, das durch hydrolytische Spaltung von 6a und anschließende Oxidation bei der Aufarbeitung gebildet wird (Tab. 1).

Tab. 1. Carbodesilylierung von (Trimethylsilyl)pyridinen $2 \mathbf{a}, 5 \mathbf{a}$ und 5 b mit Benzaldehyd

\begin{tabular}{|c|c|c|c|c|c|}
\hline Edukt & $\begin{array}{l}\text { Temp. } \\
{ }^{\circ} \mathrm{C}\end{array}$ & $\begin{array}{c}\text { Zeit } \\
h\end{array}$ & Produk & Ausb.(\%) & $\begin{array}{l}\text { Edukt }^{b)} \\
\text { Ausb.(\%) }\end{array}$ \\
\hline \multicolumn{6}{|c|}{ ohne CsF in DMF } \\
\hline $2 a$ & 100 & 3 & & & \\
\hline & 90 & $\begin{array}{c}1 \\
72\end{array}$ & $3 a$ & 3.9 & $\begin{array}{l}93.8 \\
33\end{array}$ \\
\hline $5 a$ & 130 & 8 & $\left.6 a^{c}\right)$ & - & \\
\hline $5 b$ & 130 & 8 & $\left.6 b^{c}\right)$ & - & \\
\hline \multicolumn{6}{|l|}{ mit $C s F$ in $D M F$} \\
\hline $2 a$ & $\begin{array}{l}90 \\
90\end{array}$ & $\begin{array}{c}1 \\
72\end{array}$ & $\begin{array}{l}3 a \\
3 a^{c, d)}\end{array}$ & $\begin{array}{l}2.3 \\
48\end{array}$ & $\begin{array}{l}93.4 \\
31.7\end{array}$ \\
\hline $5 a$ & $\begin{array}{l}90 \\
90 \\
90 \\
90\end{array}$ & $\begin{array}{l}1 / 4 \\
1 / 2 \\
1 \\
34\end{array}$ & $\begin{array}{l}6 a \\
6 a \\
6 a \\
\left.6 a^{c}, e\right)\end{array}$ & $\begin{array}{l}1.6 \\
4.6 \\
49\end{array}$ & $\begin{array}{l}92.4 \\
90.4 \\
89.1\end{array}$ \\
\hline $5 b$ & $\begin{array}{l}90 \\
90 \\
90 \\
90\end{array}$ & $\begin{array}{l}1 / 6 \\
1 / 2 \\
1 \\
2\end{array}$ & $\begin{array}{l}6 b \\
6 b \\
6 b \\
\left.6 b^{c}\right)\end{array}$ & $\begin{array}{l}15.6 \\
44.4 \\
56.2 \\
74\end{array}$ & $\begin{array}{l}72.9 \\
34.9 \\
12.3\end{array}$ \\
\hline
\end{tabular}

${ }^{[a]}$ Bestimmt mit GC. - ${ }^{[b]}$ Eduktkonzentration in kinetischen Messungen bestimmt. - ${ }^{\left[{ }^{c]}\right.}$ Präparative Ausb. - ${ }^{[\mathrm{d}]}$ Zusätzlich 15\% 2-

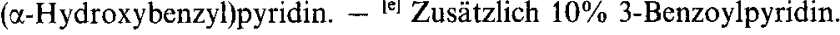

Setzt man 2 a unter gleichen Bedingungen $\left(90^{\circ} \mathrm{C}, \mathrm{DMF}\right.$, CsF) mit Benzaldehyd um, so sind selbst nach 72 Stunden noch $32 \%$ des Edukts 2 a vorhanden; neben $48 \%$ 3a werden dabei noch 15\% 2-( $\alpha$-Hydroxybenzyl)pyridin, das Hydrolyseprodukt von $\mathbf{3 a}$, isoliert, so daß eine Gesamtausbeute von $63 \%$ Carbodesilylierungsprodukt erhalten wird. Setzt man 2 a ohne Zusatz von CsF bei $90^{\circ} \mathrm{C} 72$ Stunden mit Benzaldehyd um, so werden neben $33 \%$ Edukt $66 \%$ 3a erhalten (Tab. 1).

Diese Versuche zeigen eindeutig, daß bei 2-TMS-Pyridin (2a) durch Zugabe einer starken Base (CsF) die Carbodesilylierung nicht beschleunigt wird, während dies bei 3- und

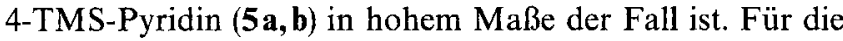
Base-katalysierten Reaktionen von $\mathbf{5} \mathbf{a}, \mathbf{b}$ ist eine pentakoordinierte Additionsverbindung und ein Pyridyl-Anion als Zwischenstufe des Reaktionsmechanismus anzunehmen ${ }^{[4,11]}$.

Aus der größeren Reaktivität von $\mathbf{5 b}$ gegenüber 5a (Tab. 1) kann man schließen, daß die Bildung des Pyridyl-
Anions in diesen Fällen geschwindigkeitsbestimmend ist, da das 4-Pyridyl-Anion stabiler als das 3-Pyridyl-Anion ist ${ }^{[12]}$.

Aus den angeführten experimentellen Befunden und Literaturangaben ${ }^{[8,9]}$ ist für die unkatalysierte Carbodesilylierung von 2a mit Benzaldehyd der in Schema 2 zusammengefaßte „Ylid-Mechanismus“ anzunehmen.

Schema 2<smiles>COC(c1ccccc1)[n+]1ccccc1</smiles><smiles></smiles>

Das primär aus Benzaldehyd mit $\mathbf{2 a}$ gebildete Additionsprodukt $\mathbf{A}$ ist selbst eine starke Base und kann über einen inter- oder intramolekularen Angriff die Trimethylsilylgruppe unter $\mathrm{Si}-\mathrm{C}$-Bindungsspaltung und unter Bildung von B, einer Zwischenstufe mit Ylid-Struktur ${ }^{[8,9,13]}$, ablösen. $\mathbf{B}$ reagiert mit einem zweiten Mol Benzaldehyd über $\mathbf{C} z u$ D weiter, das dann unter Abspaltung von Benzaldehyd das Endprodukt 3a ergibt. Ein vergleichbarer Mechanismus wurde für die Protodesilylierung von 2 a mit Wasser und Alkohol formuliert ${ }^{[13,14]}$. Das mit $\mathbf{A}$ vergleichbare Additionsprodukt aus Pyridin und Benzaldehyd konnte durch Acylierung abgefangen und nachgewiesen werden ${ }^{[15]}$.

Aus den H/D-Austauschgeschwindigkeiten in Pyridin einerseits und $N$-substituierten Pyridinium-Salzen andererscits wurde für Pyridiniumverbindungen im Vergleich $\mathrm{zu}$ Pyridin eine um den Faktor $10^{14}-10^{16}$ höhere Anionenstabilität in 2,6-Position ermittelt ${ }^{[16]}$. Da man auch beim YlidMechanismus davon ausgehen kann, daß die $\mathrm{Si}-\mathrm{C}-\mathrm{Bin}-$ dungsspaltung der geschwindigkeitsbestimmende Schritt ist, wird verständlich, daß $\mathbf{2}$ a wegen der ausgeprägten Anionenstabilisierung in $\mathbf{B}$ ausschließlich nach diesem Mechanismus reagiert, zumal das 2-Pyridyl-Anion noch instabiler als das 3-Pyridyl-Anion ${ }^{[12]}$ ist.

\section{Darstellung von 2-(Trimethylsilyl)pyridinen mit Substituenten in 4- bzw. 5-Position}

Zur weiteren Klärung des in Schema 2 postulierten Reaktionsmechanismus der Carbodesilylierung von 2-(Trimethylsilyl)pyridin (2a) mit Benzaldehyd haben wir Substituenteneinflüsse auf die Geschwindigkeit dieser Reaktion untersucht. Durch Substituenten in 4-Position von $\mathbf{2 a}$ wird sich vor allem eine mesomere Beeinflussung der Bildung von A sowie eine induktive von B bemerkbar machen. Substi- 
tuenten in 5-Position können die Bildung beider Zwischenstufen, $\mathbf{A}$ und $\mathbf{B}$, nur induktiv beeinflussen. Über die experimentell zu ermittelnden Reaktionsgeschwindigkeiten der entsprechend substituierten Pyridine sollten Aussagen über die Bedeutung der Zwischenstufen A bzw. B für diese Carbodesilylierung möglich sein. Die Auswahl der Substituenten erfolgte deshalb vor allem unter dem Gesichtspunkt, eine möglichst große Breite der mesomeren und induktiven Substituenteneffekte zu erfassen.

In der Reihe der 4- bzw. 5-substituierten 2-(Trimethylsilyl)pyridine 2 bzw. 7 wurden die Vertreter $2 \mathbf{a}-\mathbf{g}$ und $7 \mathbf{b}-\mathbf{f}$ dargestellt.

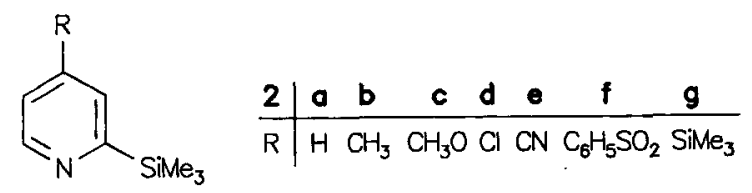

$$
\begin{aligned}
& 2 \\
& \text { Clime }
\end{aligned}
$$

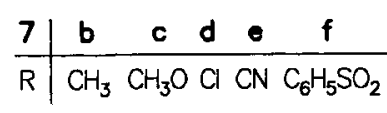

$$
\begin{aligned}
& 7
\end{aligned}
$$

Als Ausgangsverbindungen für die Einführung der TMSGruppe in 2-Position haben wir, mit Ausnahme der Phenylsulfonyl-Verbindungen $2 f$ und $7 \mathbf{f}, 4-$ bzw. 5-substituierte 2-Halogenpyridine 1 eingesetzt.

Die Methyl- und Chlor-substituierten Verbindungen $\mathbf{1 b}$, $\mathbf{b}^{\prime}$ und $\mathbf{d}^{\prime}$ können nach Craig ${ }^{[17]}$ einfach und in guten Ausbeuten aus den entsprechend substituierten 2-Aminopyridinen dargestellt werden.

Da die Phenylsulfonyl-substituierten Halogenverbindungen nicht zu $2 f$ und 7 f silyliert werden konnten, haben wir

Schema 3

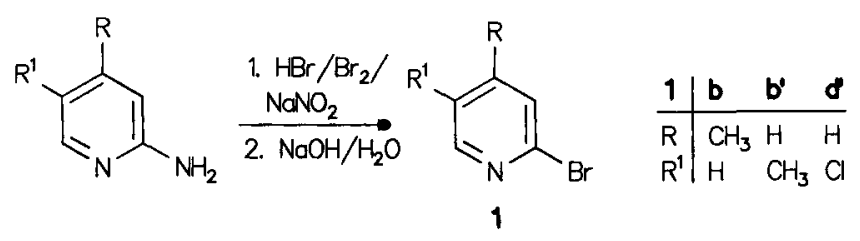

Schema 4

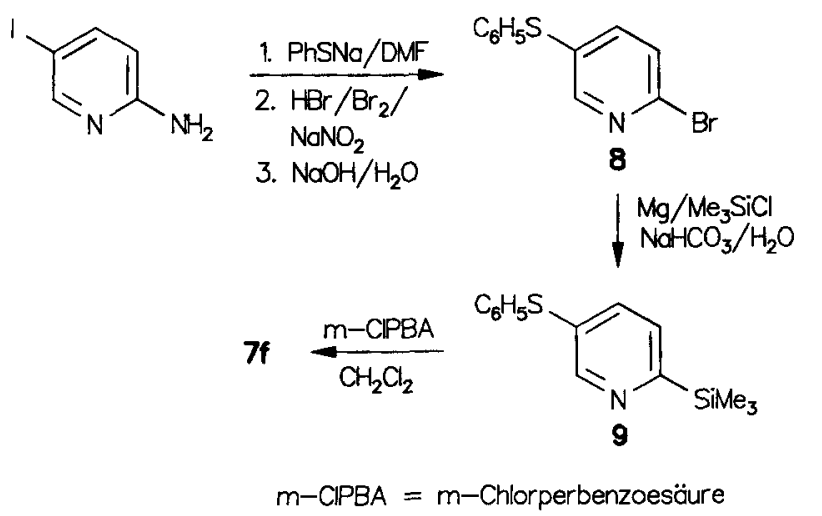

4-(Phenylsulfonyl)-2-(trimethylsilyl)pyridin (2f) durch nucleophile Substitution aus 4-Chlor-2-(trimethylsilyl)pyridin (2d) mit einem 5 fachen Überschuß an Kaliumbenzolsulfinat in Dimethylformamid hergestellt.

7f wurde durch Oxidation von 5-(Phenylthio)-2-(trimethylsilyl)pyridin (9) mit $m$-Chlorperbenzoesäure mit $26 \%$ Ausbeute erhalten (Schema 4).

Die übrigen, zum Teil bekannten, substituierten 2-Halogenpyridine 1c, $\mathbf{d}, \mathbf{e}, \mathbf{g}, \mathbf{c}^{\prime}, \mathbf{e}^{\prime}$ wurden durch Nacharbeiten oder Abwandeln bekannter Vorschriften ${ }^{[18-34]}$ in mehrstufigen Synthesen realisiert (s. Exp. Teil).

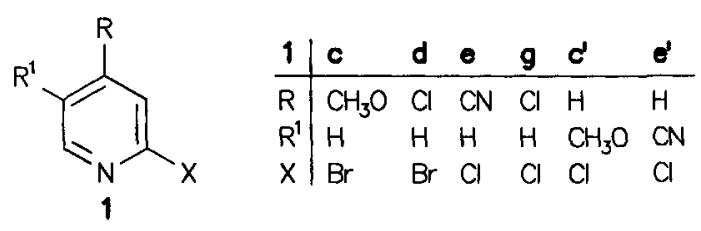

Die 2-Halogenpyridine 1 können dann problemlos in einer In-situ-Grignard-Reaktion ${ }^{[10]} \mathrm{zu}$ den 4- bzw. 5-substituierten 2-(Trimethylsilyl)pyridinen 2 bzw. 7 umgesetzt werden. Dabei wurde das üblicherweise verwendete Lösungsmittel HMPT durch Tetrahydrofuran (THF) ersetzt. Lediglich die Silylierung von 2-Chlor-5-cyanpyridin (1 $\left.\mathbf{e}^{\prime}\right) \mathrm{zu}$ 7f wurde mit Zusatz von $40 \mathrm{mmol}$ 1,2-Dibromethan (Entrainermethode ${ }^{[35]}$ ) und in HMPT als Lösungsmittel durchgeführt. Bei der Silylierung von $1 \mathrm{~g} z u \mathbf{~} \mathbf{g}$ werden 2 Moläquivalente Magnesium und Chlortrimethylsilan eingesetzt (s. Exp. Teil).

\section{Substituenteneinflüsse bei den Reaktionen der (Trimethylsilyl)pyridine 2 und 7 mit Benzaldehyd}

Die Protodesilylierung 2-Silyl-substituierter Pyridine wurde schon verschiedentlich kinetisch untersucht ${ }^{[14 a, 36]}$. Die Reaktivitätsreihenfolge ist dabei nicht einfach mit der Basizität der Pyridine korrelierbar, sondern auch die sterischen Verhältnisse am Silizium haben einen EinfluB auf die Reaktionsgeschwindigkeit. Bei einem Vergleich ${ }^{[13 b]}$ der Hydrolysegeschwindigkeiten von $\mathbf{2 a}$ und 1-Methyl-2-(trimethylsilyl)pyridinium-iodid wurde ein linearer Anstieg der Geschwindigkeit mit steigendem pH-Wert gefunden, wobei jedoch die Reaktion bei $2 \mathrm{a}$, oberhalb seines $\mathrm{p} K_{\mathrm{a}}$-Wertes von 7.07, pH-unabhängig wird. Diese Befunde sprechen dafür, $\mathrm{da} \beta \mathrm{im}$ geschwindigkeitsbestimmenden Schritt die $\mathrm{Si}-\mathrm{C}$ Bindungsspaltung des Pyridinium-Salzes durch $\mathrm{OH}^{-}$-Ionen erfolgt. Oberhalb von pH 7.07 wird bei 2 a die Beschleunigung der $\mathrm{Si}-\mathrm{C}-$ Spaltung mit zunehmender $\mathrm{OH}^{-}$-Konzentration durch die Abnahme an Pyridinium-Salz kompensiert, was $\mathrm{zu}$ einer $\mathrm{pH}$-unabhängigen Reaktionsgeschwindigkeit führt. Beim $N$-Methylpyridinium-Salz dagegen nimmt mit zunehmender $\mathrm{OH}^{-}$-Konzentration die Reaktionsgeschwindigkeit erwartungsgemäß stetig zu.

Da sich die bei früheren Untersuchungen meist angewandte UV-spektroskopische Verfolgung der Protodesilylierung ${ }^{[13 b, 14 d]}$ für unsere Untersuchungen wegen der nur geringen Unterschiede der UV-Absorptionsbanden von Edukten und Produkten als wenig geeignet erwies, wurde der Verlauf der Carbodesilylierung gaschromatographisch verfolgt. 
Die Kinetik der Reaktionen der 2-(Trimethylsilyl)pyridine 2 und 7 mit Benzaldehyd wurde als Reaktion „pseudo“erster Ordnung mit einem 20fachen Überschuß an Benzaldehyd in Dimethylformamid (DMF) ermittelt.

Unter Annahme des in Schema 2 vorgeschlagenen Reaktionsmechanismus ist folgendes Geschwindigkeitsgesetz zu formulieren:

$$
\begin{aligned}
& -\frac{\mathrm{d}[2]}{\mathrm{d} t}=k \cdot[2] \cdot[\text { Benzaldehyd }]^{n} ; \quad[\text { Benzaldehyd }]=\text { konst. } \\
& \qquad n=1,2 \\
& -\frac{\mathrm{d}[2]}{\mathrm{d} t}=k^{\prime} \cdot[2] \text { und daraus: } \ln \frac{[2]_{t=0}}{[2]}=k^{\prime} \cdot t
\end{aligned}
$$

Tab. 2. Geschwindigkeitskonstanten $k^{\prime}$ für die Umsetzungen von $2 \mathbf{a}$-f mit überschüssigem Benzaldehyd in DMF bei $55.3^{\circ} \mathrm{C}$

\begin{tabular}{c|llllll}
\hline $\mathbf{2}$ & $\mathbf{a}$ & $\mathbf{b}$ & $\mathbf{c}$ & $\mathbf{d}$ & $\mathbf{e}$ & $\mathbf{f}$ \\
\hline $\mathbf{R}$ & $\mathrm{H}$ & $\mathrm{CH}_{3}$ & $\mathrm{CH}_{3} \mathrm{O}$ & $\mathrm{Cl}$ & $\mathrm{CN}$ & $\mathrm{PhSO}_{2}$ \\
$\mathrm{~K}^{\prime}\left[\mathrm{min}^{-1}\right] \cdot 10^{-3}$ & 4.0 & 8.1 & 37.9 & 11.9 & 1.40 & 2.38 \\
Korrel.Koeff.r & 0.996 & 0.987 & 0.998 & 0.999 & 0.999 & 0.997 \\
\hline
\end{tabular}

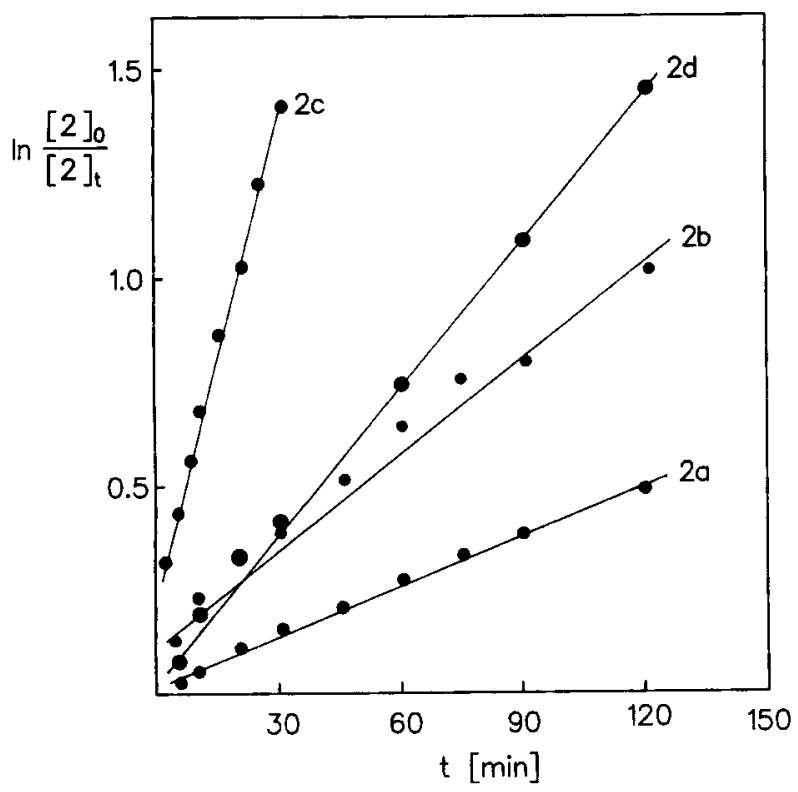

Abb. 1. Zeitabhängige Abnahme der Edukt-Konzentration bei der Umsetzung von $2 \mathrm{a}-\mathrm{d}$ mit Benzaldehyd in DMF bei $55.3^{\circ} \mathrm{C}$

Die quantitative Verfolgung der Produktbildung bei den Verbindungen $\mathbf{2 b}$ und $\mathbf{2 c}$ führt zu vergleichbaren Geschwindigkeitskonstanten, wie sie aus der Eduktabnahme erhalten werden. Da sich 2a und DMF gaschromatographisch nicht sauber trennen lassen, konnte die zeitliche Abnahme von 2a nur über das gebildete 3a errechnet werden.

Die bei den 4-substituierten Pyridinen 2 angewandten Bedingungen zur Ermittlung der Reaktionsgeschwindigkeiten wurden in analoger Weise auf die Bestimmung der Reaktionskinetiken der 5-substituierten Pyridine 7 übertragen. Dies war bei den reaktiven Vertretern $7 \mathbf{b}-\mathbf{d}$ ohne Schwie- rigkeiten möglich. Die Cyan-Verbindung 7e jedoch reagiert mit Benzaldehyd nur sehr langsam, so daß eine quantitative Erfassung der Eduktabnahme schwierig war. Die Bestim-

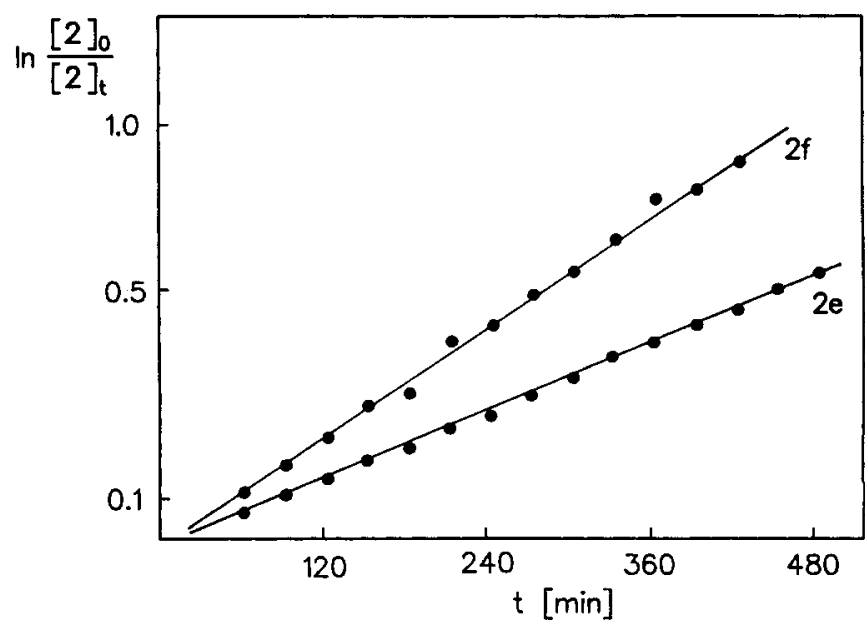

Abb. 2. Zeitabhängige Abnahme der Edukt-Konzentration bei der Umsetzung von $2 \mathrm{e}$ und $2 \mathrm{f}$ mit Benzaldehyd in DMF bei $55.3^{\circ} \mathrm{C}$

Tab. 3. Geschwindigkeitskonstanten $k^{\prime}$ für die Reaktionen von $7 \mathrm{~b}-\mathbf{e}$ mit Benzaldehyd

\begin{tabular}{c|llll}
\hline $\mathbf{7}$ & b & c & d & e \\
\hline $\mathrm{R}$ & $\mathrm{CH}_{3}$ & $\mathrm{CH}_{3} \mathrm{O}$ & $\mathrm{Cl}$ & $\mathrm{CN}$ \\
$\mathrm{k}^{\prime}\left[\mathrm{min}^{-1}\right] \cdot 10^{-3}$ & 5.5 & 1.10 & 0.52 & $\left.0.35^{\mathrm{a}}\right)$ \\
Korrel.Koeff.r & 0.999 & 0.997 & 0.997 & 0.990 \\
\hline
\end{tabular}

${ }^{[a]}$ Es wurde der Wert mit der besten Korrelation der zeitabhängigen Produktabnahme verwendet.

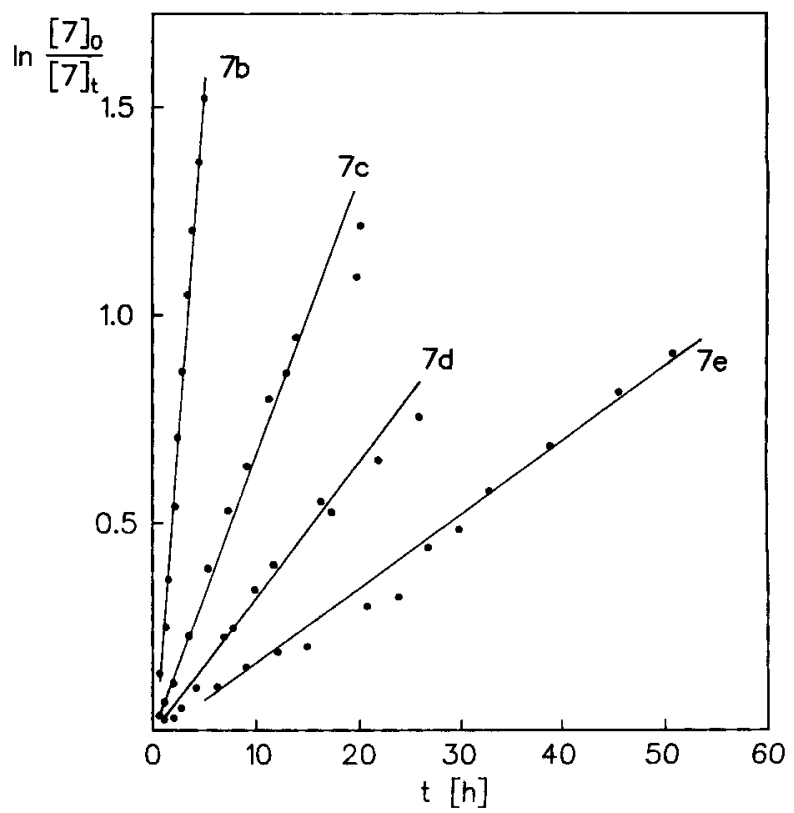

Abb. 3. Zeitabhängige Abnahme der Edukt-Konzentration bei der Umsetzung von $7 \mathbf{b}-\mathbf{e}$ mit Benzaldehyd in DMF bei $55.3^{\circ} \mathrm{C}$ 
mung der Reaktionsgeschwindigkeit der PhenylsulfonylVerbindung $7 f$ war nicht möglich.

Die kinetischen Untersuchungen haben gezeigt, daß die Reaktionsgeschwindigkeit der Carbodesilylierung der 2(Trimethylsilyl)pyridine 2 und 7 in starkem Maße von den Substituenten R im Pyridinring abhängt, wobei auch deren Position im Ring einen nicht unerheblichen Einfluß hat.

Den Einfluß der Substituenten auf die Reaktionsgeschwindigkeit der Carbodesilylierung haben wir durch Korrelation der Reaktionsgeschwindigkeiten mit den $\mathrm{p} K_{\mathrm{a}}$-Werten der Pyridine und den verschiedenen bekannten Substituentenparametern versucht zu erfassen. Da $\mathrm{p} K_{\mathrm{a}}$-Werte für die 2-(Trimethylsilyl)pyridine 2 und 7 bisher nicht beschrieben sind, wurden die Basizitäten der in 2-Position nicht silylierten Pyridine ${ }^{[37]}$ zugrundegelegt und mit den Reaktionsgeschwindigkeiten der Verbindungen 2 und 7 verglichen (Abb. 4). Dabei wird davon ausgegangen, daß die 2-TMS-Gruppe in allen Fällen einen vergleichbaren Einfluß auf die jeweiligen $\mathrm{p} K_{\mathrm{a}}$-Werte hat.

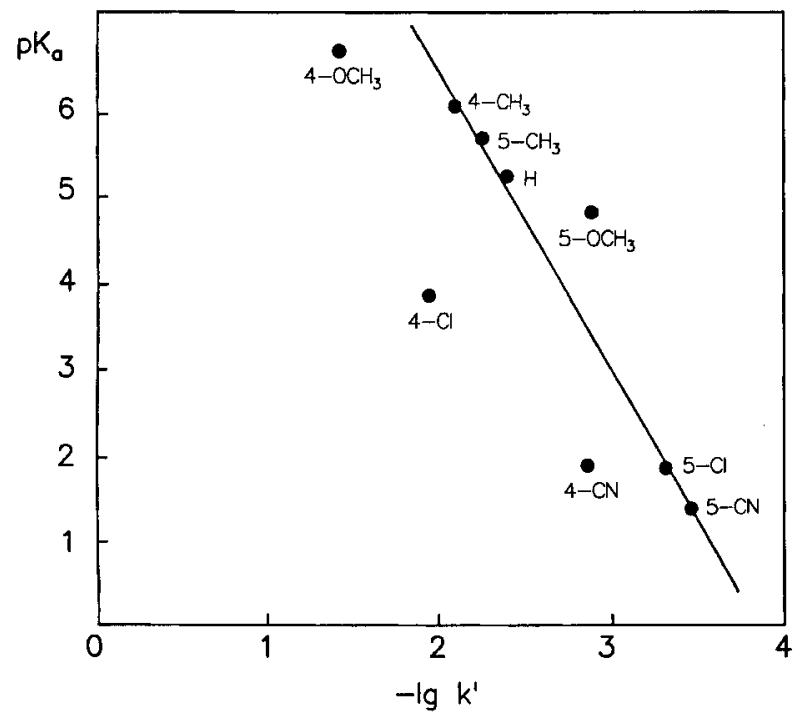

Abb. 4. Abhängigkeit der Reaktivität von 2 und 7 bei der Umsetzung mit Benzaldehyd von der Basizität der entsprechend substituierten Pyridine

Abbildung 4 zeigt, daß eine generelle Korrelation der Carbodesilylierungsgeschwindigkeit $\left(\lg k^{\prime}\right)$ mit den Basizitäten der Pyridine $\left(\mathrm{p} K_{\mathrm{a}}\right)$ nicht gegeben ist. Mit Ausnahme der 5-Methoxyverbindung 7c und der 4-Methylverbindung 2b besteht jedoch eine gute lineare Beziehung zwischen Reaktivität und $\mathrm{p} K_{\mathrm{a}}$-Werten bei den 5-substituierten Verbindungen 7 einerseits und den 4-substituierten Verbindungen 2 andererseits. Für die Abweichungen der beiden Vertreter könnten die unterschiedlichen Lösungsmittelsysteme bei der $\mathrm{p} K_{\mathrm{a}}$-Wert-Bestimmung (Wasser) und bei der Carbodesilylierung (DMF) verantwortlich sein, da diese die elektronischen Eigenschaften der Verbindungen in starkem Maße beeinflus$\operatorname{sen}^{[38]}$.

Nachdem eine Korrelation der Reaktionsgeschwindigkeiten mit der Basizität zu keinem befriedigenden Ergebnis führte, haben wir Vergleiche mit den auf Basis der „Linearen
Freien Enthalpie-Beziehung (LFER)“ ermittelten Substituentenparametern angestellt. Die uneingeschränkte Verwendbarkeit von LFER-Parametern in Pyridinen - auch bei Reaktionen am Heteroatom - wurde mehrfach aufgezeigt ${ }^{[37,39]}$. Bei Siliciumverbindungen war für die Protodesilylierung von Arylsilanen mit Säure ${ }^{[40]}$ eine gute Korrelation der Reaktionsgeschwindigkeit mit $\sigma^{+}$-Werten, für die basekatalysierte Proto- bzw. Carbodesilylierung eine befriedigende Korrelation mit $\sigma$ - bzw. $\sigma^{0}$-Konstanten gefunden worden ${ }^{[4,41]}$.

Für die untersuchte Carbodesilylierung ergab sich eine gute Korrelation mit der von Taft et al. ${ }^{[42]}$ entwickelten Dual-Parameter-Gleichung (G1. 1) unter Verwendung bekannter $\sigma_{R}^{0}$ - und $\sigma_{I}-$ Werte $^{[43]}$. Es ergaben sich die Gleichungen (2) und (3) für die 4-substituierten Pyridine $2 \mathbf{a}-\mathbf{f}$ und die 5-substituierten Vertreter $7 \mathbf{b}-\mathbf{e}$.

$$
\begin{aligned}
\mathrm{P}-\mathrm{P}_{0}= & \mathrm{Q}_{\mathrm{I}} \sigma_{\mathrm{I}}+\mathrm{Q}_{\mathrm{R}} \sigma_{\mathrm{R}} \\
\Delta \lg k_{4 \mathrm{R}}^{\prime}= & 0.23 \cdot \sigma_{\mathrm{I}}+2.65 \cdot \sigma_{\mathrm{R}}^{0} \\
& (n=5, s=1.37) \\
\Delta \lg k_{S \mathrm{R}}^{\prime}= & 1.94 \cdot \sigma_{\mathrm{I}}+0.18 \cdot \sigma_{\mathrm{R}}^{0} \\
& (n=4, s=2.55)
\end{aligned}
$$

Nach Lit. ${ }^{[42 b]}$ ist es wahrscheinlich, daß $\mathrm{Q}_{\mathrm{I}}$-Werte mit $\varrho_{R} / \varrho_{I} \geqslant 10$ bzw. $Q_{R}-$ Werte mit $\varrho_{I} / \varrho_{R} \geqslant 10$ Artefakte sind und vernachlässigt werden können. Unter dieser Annahme vereinfachen sich Gl. (2) zu Gl. (4) und Gl. (3) zu Gl. (5).

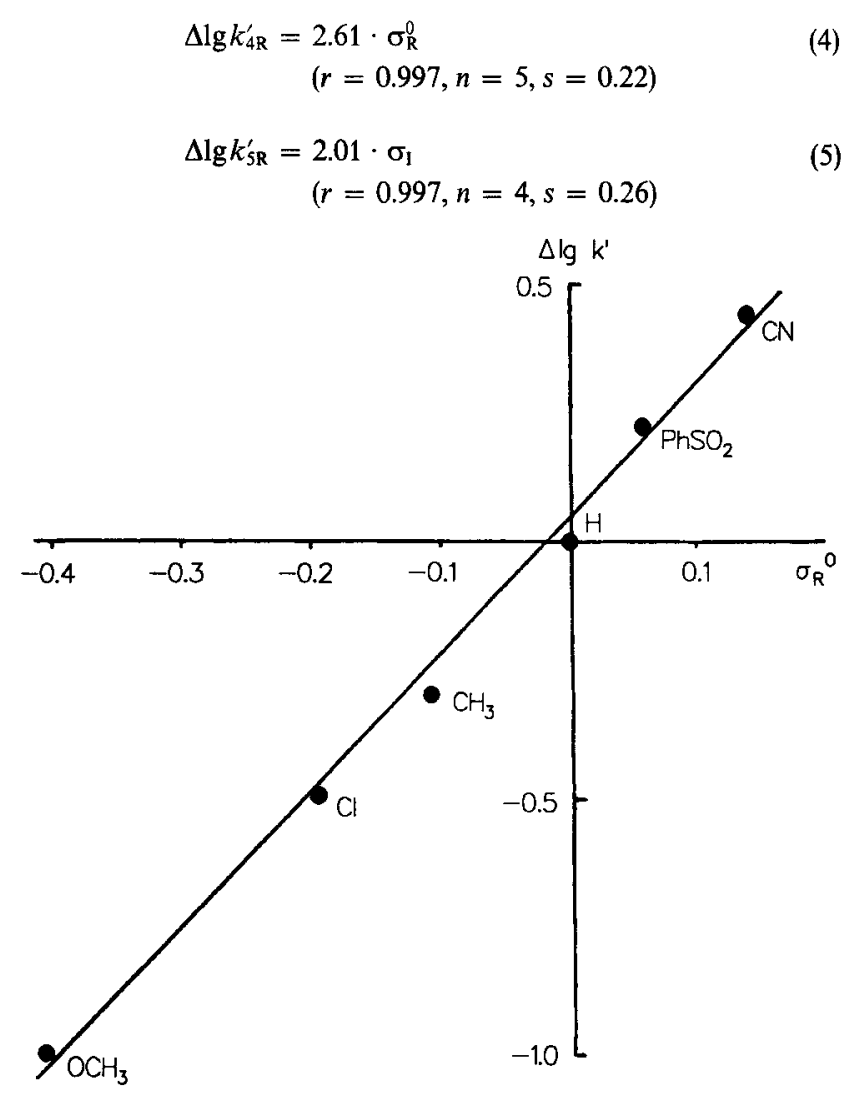

Abb. 5. Korrelation der $\Delta \lg k^{\prime}$-Werte von $2 \mathbf{a}-\mathbf{f}$ mit $\sigma_{\mathbf{R}}^{0}$ 


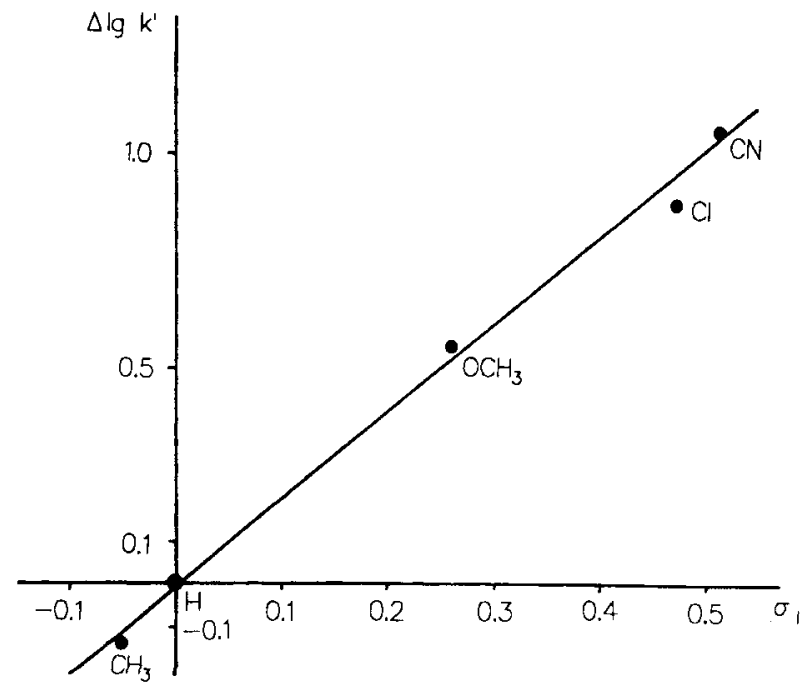

Abb. 6. Korrelation der $\Delta \lg k^{\prime}-$ Werte von $2 \mathrm{a}$ und $7 \mathbf{b}-\mathbf{e}$ mit $\sigma_{\mathrm{I}}$

Tab. 4. $\sigma_{1}$ und $\sigma_{R}^{0}$-Werte der verschiedenen Substituenten $\mathrm{R}$ nach Lit. ${ }^{[43]}$ und Desilylierungsgeschwindigkeitskonstanten $\lg k^{\prime}$ der Verbindungen 2 und 7

\begin{tabular}{|c|c|c|c|c|c|c|}
\hline $\mathrm{R}$ & $\sigma_{1}$ & $\sigma_{R}^{0}$ & \multicolumn{4}{|c|}{$\begin{array}{l}\Delta l g k^{\prime}=\lg k_{R}^{\prime}-\lg k_{H}^{\prime} \\
\text { Verbind. } 2\end{array}$} \\
\hline $\begin{array}{l}\mathrm{H} \\
\mathrm{CH}_{3} \\
\mathrm{CH}_{3} \mathrm{O} \\
\mathrm{Cl} \\
\mathrm{CN} \\
\mathrm{C}_{6} \mathrm{H}_{5} \mathrm{SO}_{2}\end{array}$ & $\begin{array}{l}0 \\
-0.05 \\
0.26 \\
0.47 \\
0.52 \\
0.55\end{array}$ & $\begin{array}{l}0 \\
-0.10 \\
-0.41 \\
-0.20 \\
0.14 \\
0.06\end{array}$ & $\begin{array}{l}2 a \\
2 b \\
2 c \\
2 d \\
2 e \\
2 f\end{array}$ & $\begin{array}{l}0 \\
-0.31 \\
-0.98 \\
-0.48 \\
0.45 \\
0.22\end{array}$ & $\begin{array}{l}7 b \\
7 c \\
7 d \\
7 e \\
7 f\end{array}$ & $\begin{array}{r}-0.14 \\
0.56 \\
0.88 \\
1.06\end{array}$ \\
\hline
\end{tabular}

Die Abbildungen 5 und 6 bestätigen die sehr gute Korrelation zwischen der Reaktionsgeschwindigkeit der Carbodesilylierung 2-TMS-substituierter Pyridine mit dem mesomeren Einfluß von Substituenten in 4-Position und dem induktiven Einfluß von Substituenten in 5-Position.

Da die Bestimmung von $\sigma_{\mathrm{R}}$-Werten bisher nur durch indirekte Methoden möglich und sie deshalb auch mit gröBeren Fehlern behaftet ist als die Bestimmung der $\sigma_{\mathrm{I}}$-Werte, könnte über den hier beschriebenen Weg eine direkte experimentelle Ermittlung von $\sigma_{\mathrm{R}}$-Werten erfolgen.

Aufgrund der experimentell ermittelten Substituenteneinflüsse auf die Geschwindigkeit der Carbodesilylierung von 2-TMS-Pyridinen kann der in Schema 2 vorgeschlagene Reaktionsmechanismus präzisiert werden.

Eine Stabilisierung des Pyridinium-Salzes $\mathbf{A}$ ist durch $+\mathrm{M}$ - und + I-Substituenten zu erwarten, und zwar sowohl von Substituenten in 4- als auch in 5-Position. Bei der YlidZwischenstufe B ist - wegen der Orthogonalität des Anionen-Orbitals - jedoch nur eine induktive Beeinflussung der Stabilität des Anions möglich, wobei die induktiven Einflüsse von Substituenten in 4- bzw. 5-Position als vergleichbar angenommen werden können. Man kann bei $\mathbf{B}$ weiter davon ausgehen, daß sich die induktiven Einflüsse von Substituenten bei der Stabilisierung bzw. Destabilisierung von positiver und negativer Ladung kompensieren.

Diese Annahme wird gestützt durch den Befund, daß bei 3-substituierten Pyridinen in neutraler Lösung kein Substi- tuenteneinfluß auf die $\mathrm{H} / \mathrm{D}$-Austauschgeschwindigkeit festgestellt wird ${ }^{[44 a]}$, da sich die induktiven Einflüsse für die Bildung des Pyridiniumsalzes bzw. des Ylids gegenseitig kompensieren. Pyridin- $N$-oxide, bei denen die Pyridiniumstruktur nicht in einem Vorgleichgewicht gebildet werden muB, zeigen dagegen die erwartete lineare Abhängigkeit der $\mathrm{H} / \mathrm{D}$-Austauschgeschwindigkeit von den $\sigma_{\mathrm{I}}$-Werten der zur negativen Ladung benachbarten Substituenten in 3-Position $^{[44 b]}$.

Aus der deutlichen Abhängigkeit der Carbodesilylierungsgeschwindigkeit vom induktiven Einfluß der 5-Substituenten in $\mathbf{7 b}-\mathbf{e}$ schließen wir deshalb, daß die Konzentration von $\mathbf{A}$ für die Geschwindigkeit der Produktbildung entscheidend ist und nicht die Bildung von $\mathbf{B}$ aus $\mathbf{A}$, da sich wie oben ausgeführt - der induktive Einfluß eines 5-Substituenten auf die Stabilität von B nicht auswirken sollte, wohl aber auf die Konzentration von $\mathbf{A}$.

Wir danken Frau G. Mündl für die Darstellung von 5-substituierten 2-Halogenpyridinen und dem Fonds der Chemischen Industrie für die Unterstützung dieser Arbeit.

\section{Experimenteller Teil}

${ }^{1}$ H-NMR: Varian T 60 sowie Bruker WP 80 und CXP 300, TMS als interner Standard. - GC: a) Hewlett-Packard $5700 \mathrm{~A}$ und 5710 A mit FID, Spectra Physics Minigrator; Trägergas: Reinstickstoff; Glassäulen, $2.3 \mathrm{~m} \times 2 \mathrm{~mm}$, Phasen: OV 17, 101, 225 und Carbowax M20 auf Chromosorb W. b) Carlo Erba Fractovap GI 4160 mit FID, Spectra Physics Minigrator; Trägergas: 0.7 bar Helium; Glaskapillare, $20 \mathrm{~m}$, Phase: SE 54. - Präparative Säulenchromatographie: Glassäulen verschiedener Größe, gepackt mit Kieselgel S, Korngröße 0.032-0.063 mm (Riedel de Haen), Aluminiumoxid Neutral Super I (ICN Biomedicals).

Darstellung der 4- und 5-substituierten 2-Brompyridine $\mathbf{1} \mathbf{b}, \mathbf{b}^{\prime}$ und

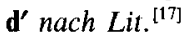

a) 2-Brom-4-methylpyridin (1 b): Aus $54.1 \mathrm{~g}$ (0.5 mol) 2-Amino-4methylpyridin, $248.0 \mathrm{ml}$ (2.2 mol) 48proz. Bromwasserstoffsäure $(\mathrm{HBr}), 239.7 \mathrm{~g}(1.5 \mathrm{~mol})$ Brom, $86.3 \mathrm{~g}(1.25 \mathrm{~mol})$ Natriumnitrit in $130 \mathrm{ml}$ Wasser und $188.5 \mathrm{~g}(4.7 \mathrm{~mol})$ Natriumhydroxid in $500 \mathrm{ml}$ Wasser, Ausb. $77.1 \mathrm{~g}(90 \%) \mathbf{1 b}$, Sdp. $90^{\circ} \mathrm{C} / 8$ Torr (Lit. ${ }^{[17 b]}$ $100-105^{\circ} \mathrm{C} / 8$ Torr).

b) 2-Brom-5-methylpyridin (1 b'): Aus $20.0 \mathrm{~g}(0.18 \mathrm{~mol})$ 2-Amino5-methylpyridin, $93 \mathrm{ml}(0.82 \mathrm{~mol}) 48$ proz. $\mathrm{HBr}, 27.7 \mathrm{ml}(0.54 \mathrm{~mol})$ Brom, $31.9 \mathrm{~g}(0.46 \mathrm{~mol}) \mathrm{NaNO}_{2}$ in $48 \mathrm{ml}$ Wasser und $70.0 \mathrm{~g}(1.75$ $\mathrm{mol}) \mathrm{NaOH}$ in $150 \mathrm{ml}$ Wasser, Ausb. $27.2 \mathrm{~g} \mathrm{(88 \% )} 1 \mathbf{b}^{\prime}$.

c) 2-Brom-5-chlorpyridin (1 d $)$ : Aus $46.0 \mathrm{~g}(0.36 \mathrm{~mol})$ 2-Amino-5chlorpyridin, $235 \mathrm{~g}$ (1.40 mol) 48proz. $\mathrm{HBr}, 73.6 \mathrm{ml}$ (1.4 mol) Brom, $84.3 \mathrm{~g}(1.22 \mathrm{~mol}) \mathrm{NaNO}_{2}$ in $125 \mathrm{ml}$ Wasser und $184 \mathrm{~g}(4.6 \mathrm{~mol})$ $\mathrm{NaOH}$ in $500 \mathrm{ml}$ Wasser, Ausb. $55 \mathrm{~g}(79 \%) 1 \mathrm{~d}^{\prime}$, Schmp. $70^{\circ} \mathrm{C}$ (Methanol) [Lit. ${ }^{[17 b]} 70-71^{\circ} \mathrm{C}$ (Methanol)].

\section{2-Brom-4-methoxypyridin (1 c) nach Lit. ${ }^{[18,20,21]}$}

a) 2-Brompyridin- $N$-oxid: Nach Lit. ${ }^{[18]}$, jedoch $3 \mathrm{~d}$ bei Raumtemp., dreimalige Extraktion mit je $200 \mathrm{ml} 15$ proz. Schwefelsäure, Zugabe von Thiosulfat, Neutralisation mit Natriumcarbonat und dreimalige Extraktion mit je $200 \mathrm{ml}$ Chloroform; aus $163.0 \mathrm{~g} \mathrm{(1.03}$ $\mathrm{mol}$ ) 2-Brompyridin, $178.0 \mathrm{~g}(1.3 \mathrm{~mol})$ Perbenzoesäure in $1.81 \mathrm{Chlo-}$ roform, Rohausb. $163.5 \mathrm{~g} \mathrm{(91 \% )} \mathrm{2-Brompyridin-} N$-oxid, ohne Reinigung weiter umgesetzt. 
b) 2-Brom-4-nitropyridin- $N$-oxid: $\mathrm{Zu} 400 \mathrm{ml} 96$ proz. Schwefelsäure werden bei $0^{\circ} \mathrm{C} 150.0 \mathrm{~g}(0.86 \mathrm{~mol}) 2$-Brompyridin- $N$-oxid, anschließend $140 \mathrm{ml} 96 \mathrm{proz}$. Salpetersäure gegeben. Nach 3stdg. Erhitzen auf $130^{\circ} \mathrm{C}$ wird das Reaktionsgemisch auf Eis gegossen und das Produkt durch Zugabe von Natriumcarbonat ausgefällt, Ausb. 145.2 g (77\%) 2-Brom-4-nitropyridin- $N$-oxid, Schmp. $145^{\circ} \mathrm{C}$ (Lit. ${ }^{[19]} 145.5-146^{\circ} \mathrm{C}$ ).

c) 2-Brom-4-nitropyridin: Nach Lit. ${ }^{[20]}$, aus $40.0 \mathrm{~g}(0.2 \mathrm{~mol}) 2-$ Brom-4-nitropyridin- $N$-oxid, $78.0 \mathrm{~g}(0.3 \mathrm{~mol})$ Phosphortribromid, Ausb. $30.0 \mathrm{~g} \mathrm{(74 \% )} \mathrm{2-Brom-4-nitropyridin,} \mathrm{Schmp.} 61-63^{\circ} \mathrm{C}$ (Lit. ${ }^{[201} 64^{\circ} \mathrm{C}$ ).

d) $1 \mathrm{c}$ : Nach Lit. ${ }^{[21]}$, aus $18.8 \mathrm{~g}(93.0 \mathrm{mmol})$ 2-Brom-4-nitropyridin, $3.2 \mathrm{~g}(140.0 \mathrm{mmol})$ Natrium in $200 \mathrm{ml}$ Methanol, Ausb. $16.0 \mathrm{~g}$ $(92 \%) 1 \mathrm{c}, \mathrm{Sdp} .116-118^{\circ} \mathrm{C} / 8$ Torr (Lit. ${ }^{[21]} 120-121^{\circ} \mathrm{C} / 10$ Torr).

\section{2-Brom-4-chlorpyridin (1d) nach Lit. ${ }^{[22-24]}$}

a) 4-Amino-2-brompyridin: Nach Lit. ${ }^{[22]}$, jedoch nach Beendigung der Reaktion Entfernen der Essigsäure und Alkalisieren; aus $40.0 \mathrm{~g}$ $(0.18 \mathrm{~mol}) 2$-Brom-4-nitropyridin- $N$-oxid, $30.0 \mathrm{~g}(0.54 \mathrm{~mol})$ Eisenpulver und $300 \mathrm{ml}$ Essigsäure, Ausb. $28.0 \mathrm{~g}$ (90\%) 4-Amino-2brompyridin, Schmp. $94-96^{\circ} \mathrm{C}$ (Lit. ${ }^{[19]} 95-97^{\circ} \mathrm{C}$ ).

b) 2-Brom-4-(nitroamino) pyridin: Nach Lit. ${ }^{[23]}$, jedoch Zugabe der Salpetersäure bei -5 bis $-10^{\circ} \mathrm{C}$; aus $8.40 \mathrm{~g}$ (48.5 mmol) 4Amino-2-brompyridin, $90 \mathrm{ml}$ 96proz. Schwefelsäure und $45 \mathrm{ml}$ 96proz. Salpetersäure, Ausb. $8.31 \mathrm{~g}(79 \%)$ 2-Brom-4-(nitroamino)pyridin, Schmp. $180-181^{\circ} \mathrm{C}$ (Zers.) (Lit. ${ }^{[23]} 181^{\circ} \mathrm{C}$ ).

c) $1 \mathrm{~d}$ : Nach Lit. ${ }^{[24]}$, aus $21.1 \mathrm{~g}(96.8 \mathrm{mmol}) 2$-Brom-4-(nitroamino)pyridin, $40.0 \mathrm{~g}$ (192.1 mmol) Phosphorpentachlorid und $140 \mathrm{ml}$ Chloroform, Ausb. 12.0 g (65\%) 1d, Sdp. $83-85^{\circ} \mathrm{C} / 8$ Torr (Lit. ${ }^{[24]}$ $98^{\circ} \mathrm{C} / 15$ Torr).

\section{2-Chlor-4-cyanpyridin (1 e) nach Lit. ${ }^{[25,26]}$}

a) Isonicotinamid: Nach Lit. ${ }^{[25]}$, aus $76.9 \mathrm{~g}(0.56 \mathrm{~mol})$ Isonicotinsäure-methylester und $135 \mathrm{ml}$ Ammoniak, Ausb. $60.0 \mathrm{~g} \mathrm{(88 \% )} \mathrm{Iso-}$ nicotinamid.

b) Isonicotinamid-N-oxid: Nach Lit. ${ }^{[26]}$, aus $75 \mathrm{~g}(0.61 \mathrm{~mol})$ Isonicotinamid, $120 \mathrm{ml} \mathrm{30proz.} \mathrm{Wasserstoffperoxid} \mathrm{und} 750 \mathrm{ml}$ Essigsäure, Ausb. $64.6 \mathrm{~g}(76 \%)$ Isonicotinamid- $N$-oxid.

c) 1e: Nach Lit. ${ }^{[26]}$, aus $32.5 \mathrm{~g}(0.24 \mathrm{~mol})$ Isonicotinamid- $N$-oxid, $70 \mathrm{~g}(0.34 \mathrm{~mol})$ Phosphorpentachlorid und $105 \mathrm{ml}$ (1.15 mol) Phosphoroxychlorid, Ausb. $20 \mathrm{~g}(60 \%) 1 \mathrm{e}$.

4-(Phenylsulfonyl)-2-(trimethylsilyl)pyridin (2f): Man erhitzt $0.37 \mathrm{~g}(2.0 \mathrm{mmol}) 2 \mathrm{~d}$ und $1.80 \mathrm{~g}(10.0 \mathrm{mmol})$ Kaliumbenzolsulfinat in $15 \mathrm{ml}$ Dimethylformamid $16 \mathrm{~h}$ auf $90^{\circ} \mathrm{C}$. Die Lösung versetzt man mit Wasser, extrahiert mehrmals mit Diethylether, trocknet die vereinigten Etherextrakte mit Natriumsulfat und engt im Rotationsverdampfer ein. Der Rückstand wird über Aluminiumoxid mit Petrolether/Ethylacetat (2:1) chromatographiert, Ausb. $0.28 \mathrm{~g}$ (48\%) 2f, Schmp. 83.5-84.5 ${ }^{\circ} \mathrm{C}$. $-{ }^{1} \mathrm{H}-\mathrm{NMR}\left(\mathrm{CDCl}_{3}\right): \delta=0.34[\mathrm{~s}$, $\left.9 \mathrm{H}, \mathrm{Si}\left(\mathrm{CH}_{3}\right)_{3}\right], 7.5-7.7(\mathrm{~m}, 4 \mathrm{H}, \mathrm{Ph}), 7.9-8.0(\mathrm{~m}, 3 \mathrm{H}, 3-, 5-\mathrm{H}, \mathrm{Ph})$, $8.96\left(\mathrm{dd}, J_{1}=5.3, J_{2}=1.0 \mathrm{~Hz}, 1 \mathrm{H}, 6-\mathrm{H}\right)$.

$$
\begin{aligned}
& \mathrm{C}_{14} \mathrm{H}_{17} \mathrm{NO}_{2} \mathrm{SSi} \text { (291.4) } \\
& \text { Ber. C } 57.70 \text { H } 5.88 \text { N } 4.81 \text { S } 11.01 \\
& \text { Gef. C } 57.58 \text { H } 6.03 \text { N } 4.87 \text { S } 10.87
\end{aligned}
$$

2,4-Dichlorpyridin (1 g): Nach Lit. ${ }^{[27]}$ aus $1.40 \mathrm{~g}(10 \mathrm{mmol}) 4-\mathrm{Ni}$ tropyridin- $N$-oxid und $4.60 \mathrm{~g}(30 \mathrm{mmol})$ Phosphoroxychlorid, Ausb. $0.46 \mathrm{~g} \mathrm{(31 \% )} 1 \mathrm{~g}$.

\section{2-Chlor-5-methoxypyridin (1 $\left.\mathbf{c}^{\prime}\right)$ nach Lit. ${ }^{[28-30]}$}

a) 5-Nitro-2(1H)-pyridinon: Nach Lit. ${ }^{[28 \mathrm{a}]}$, aus $100.0 \mathrm{~g}(0.72 \mathrm{~mol})$ 2-Amino-5-nitropyridin, $750 \mathrm{ml}$ Wasser, $150 \mathrm{ml}$ 96proz. Schwefel- säure und $70.0 \mathrm{~g}(1.0 \mathrm{~mol}) \mathrm{NaNO}_{2}$, Rohausb. $100 \mathrm{~g}(99 \%)$ 5-Nitro$2(1 H)$-pyridinon, ohne Reinigung weiter umgesetzt.

b) 2-Chlor-5-nitropyridin: Nach Lit. ${ }^{[28 \mathrm{~b}}$, aus $100 \mathrm{~g}$ (0.71 mol) 5Nitro-2(1H)-pyridinon, $160 \mathrm{~g}(0.78 \mathrm{~mol})$ Phosphorpentachlorid, $4.5 \mathrm{ml}$ ( $50 \mathrm{mmol}$ ) Phosphoroxychlorid, Ausb. $92.3 \mathrm{~g} \mathrm{(82 \% )} \mathrm{2-Chlor-}$ 5-nitropyridin, Schmp. $109^{\circ} \mathrm{C}$ (Lit. ${ }^{\text {[28b }} 107-108^{\circ} \mathrm{C}$ ).

c) 5-Amino-2-chlorpyridin: Nach Lit. ${ }^{[29]}$, aus $15.8 \mathrm{~g}(0.1 \mathrm{~mol}) 2-$ Chlor-5-nitropyridin, $95.0 \mathrm{~g}(0.5 \mathrm{~mol})$ Zinnchlorid und $150 \mathrm{ml}$ konz. Salzsäure, Ausb. 12.0 g (93\%) 5-Amino-2-chlorpyridin.

d) 2-Chlor-5-hydroxypyridin: Nach Lit. ${ }^{\text {[30a] }}$, jedoch ohne Zugabe von Kupfersulfat; aus $7.71 \mathrm{~g} \mathrm{(60.0} \mathrm{mmol)} \mathrm{5-Amino-2-chlorpyridin,}$ $4.20 \mathrm{~g}(60 \mathrm{mmol}) \mathrm{NaNO}_{2}, 211 \mathrm{~N} \mathrm{H}_{2} \mathrm{SO}_{4}, 200 \mathrm{ml} 2 \mathrm{~N} \mathrm{H}_{2} \mathrm{SO}_{4}$, Rohausb. $2.50 \mathrm{~g} \mathrm{(32 \% )} \mathrm{2-Chlor-5-hydroxypyridin,} \mathrm{ohne} \mathrm{Reinigung} \mathrm{wei-}$ ter umgesetzt.

e) $1 \mathbf{c}^{\prime}:$ Nach Lit. ${ }^{[30 b]}$, jedoch bei der Aufarbeitung mit Diethylether ausschütteln, Extrakte mit Natriumsulfat trocknen, einengen und den Rückstand destillieren; aus $2.50 \mathrm{~g}$ (19.3 mmol) 2-Chlor-5-hydroxypyridin, $0.44 \mathrm{~g}(19.3 \mathrm{mmol})$ Natrium in $5.0 \mathrm{ml}$ Methanol, $20 \mathrm{ml}$ Dimethylsulfoxid und $2.74 \mathrm{~g}(19.3 \mathrm{mmol})$ Methyliodid, Ausb. $1.17 \mathrm{~g}(32 \%) 1 \mathbf{c}^{\prime}$, Sdp. $110^{\circ} \mathrm{C} / 8$ Torr.

\section{2-Chlor-5-cyanpyridin $\left(\mathbf{1} \mathbf{e}^{\prime}\right)$ nach Lit. $^{[31,32]}$}

a) 6-Hydroxynicotinsäure: Nach Lit. ${ }^{[31]}$, aus $80 \mathrm{~g}(0.51 \mathrm{~mol}) \mathrm{Cu}-$ malinsäure-methylester, $208 \mathrm{ml} \mathrm{14proz}$. Ammoniumhydroxid-Lösung, $1066 \mathrm{ml} 17 \mathrm{proz}$. Natronlauge und $420 \mathrm{ml} \mathrm{konz}$. Salzsäure, Ausb. 45 g (64\%) 6-Hydroxynicotinsäure, Schmp. $298^{\circ} \mathrm{C}$ (Lit. ${ }^{[31]}$ $299-300^{\circ} \mathrm{C}$ ).

b) 6-Chlornicotinamid: Nach Lit. ${ }^{[32]}$, aus $45 \mathrm{~g}(0.32 \mathrm{~mol}) 6-\mathrm{Hy}-$ droxynicotinsäure, $75 \mathrm{ml}(0.82 \mathrm{~mol})$ Phosphoroxychlorid, $75 \mathrm{ml}$ Aceton und $150 \mathrm{ml}$ Ammoniak, Ausb. $15 \mathrm{~g} \mathrm{(30 \% )} \mathrm{6-Chlornicotin-}$

\begin{tabular}{|c|c|c|c|c|c|c|c|}
\hline \multicolumn{2}{|c|}{$\begin{array}{l}\text { Edukte } \\
\mathrm{g}(\mathrm{mol})\end{array}$} & \multirow{2}{*}{$\begin{array}{l}\begin{array}{l}\text { THF } \\
\mathrm{ml}\end{array} \\
400\end{array}$} & \multirow{2}{*}{$\begin{array}{c}\begin{array}{c}\text { Magnesium } \\
\mathrm{g} \text { (gat) }\end{array} \\
\begin{array}{c}29.2 \\
(1.2)\end{array}\end{array}$} & \multirow{2}{*}{$\begin{array}{l}\text { TMCS } \\
\mathrm{g}(\mathrm{mol}) \\
\begin{array}{l}130.2 \\
(1.2)\end{array}\end{array}$} & \multirow{2}{*}{\multicolumn{2}{|c|}{$\begin{aligned} \begin{array}{r}\text { Produkte } \\
\text { Ausb } \\
\mathrm{g}(\%)\end{array} \\
2 b \begin{array}{l}109.2 \\
(73)\end{array}\end{aligned}$}} & \multirow{2}{*}{$\begin{array}{c}\begin{array}{c}\text { Sdp. } \\
{\left[{ }^{\circ} \mathrm{C} / \text { Torr }\right]}\end{array} \\
74-76 / 12\end{array}$} \\
\hline 1b & $\begin{array}{l}160.0 \\
(0.9)\end{array}$ & & & & & & \\
\hline 1c & $\begin{array}{l}16.8 \\
(0.09)\end{array}$ & 80 & $\begin{array}{l}2.1 \\
(0.08)\end{array}$ & $\begin{array}{l}9.3 \\
(0.08)\end{array}$ & $2 c$ & $\begin{array}{l}7.2 \\
(44)\end{array}$ & $109-112 / 12$ \\
\hline 1d & $\begin{array}{l}17.5 \\
(0.09)\end{array}$ & 250 & $\begin{array}{l}2.4 \\
(0.1)\end{array}$ & $\begin{array}{l}10.9 \\
(0.1)\end{array}$ & $2 d$ & $\begin{array}{l}4.9 \\
(30)\end{array}$ & $87-88.5 / 12$ \\
\hline $1 e$ & $\begin{array}{l}13.80 \\
(0.1)\end{array}$ & 300 & $\begin{array}{l}3.04 \\
(0.125)\end{array}$ & $\begin{array}{l}16.30 \\
(0.15)\end{array}$ & $2 e$ & $\begin{array}{l}7.75 \\
(44)\end{array}$ & $68-70 / 0.1$ \\
\hline $1 \mathrm{~g}$ & $\begin{array}{l}1.3 \\
(0.01)\end{array}$ & 25 & $\begin{array}{l}0.5 \\
(0.02)\end{array}$ & $\begin{array}{l}2.20 \\
(0.02)\end{array}$ & $2 g$ & $\begin{array}{l}0.5 \\
(22)\end{array}$ & $85-90 / 12$ \\
\hline 1b' & $\begin{array}{l}23.7 \\
(0.15)\end{array}$ & 200 & $\begin{array}{l}4.40 \\
(0.18)\end{array}$ & $\begin{array}{l}21.7 \\
(0.20)\end{array}$ & $7 b$ & $\begin{array}{l}16.5 \\
(67)\end{array}$ & $77 / 9$ \\
\hline $1 c^{\prime}$ & $\begin{array}{l}6.89 \\
(0.048)\end{array}$ & 70 & $\begin{array}{l}1.46 \\
(0.06)\end{array}$ & $\begin{array}{l}6.48 \\
(0.06)\end{array}$ & $7 c$ & $\begin{array}{l}1.59 \\
(19)\end{array}$ & $92 / 8$ \\
\hline 1d' & $\begin{array}{l}10.0 \\
(0.052)\end{array}$ & 42 & $\begin{array}{l}1.25 \\
(0.051)\end{array}$ & $\begin{array}{l}6.65 \\
(0.061)\end{array}$ & $7 d$ & $\begin{array}{l}4.4 \\
(46)\end{array}$ & $76-78 / 9$ \\
\hline $1 e^{\prime}$ & $\begin{array}{l}\left.6.80^{a}\right)^{\prime} \\
(0.040)\end{array}$ & $60^{b)}$ & $\begin{array}{l}2.43 \\
(0.10)\end{array}$ & $\begin{array}{l}10.61 \\
(0.10)\end{array}$ & $7 e$ & $\begin{array}{l}0.85 \\
(12)\end{array}$ & $65 / 0.0005$ \\
\hline 8 & $\begin{array}{l}6.65 \\
(0.020)\end{array}$ & 60 & $\begin{array}{l}1.22 \\
(0.050)\end{array}$ & $\begin{array}{l}5.77 \\
(0.055)\end{array}$ & 9 & $\begin{array}{l}2.0 \\
\text { (31) }\end{array}$ & $95-100 / 0.001$ \\
\hline
\end{tabular}
amid, Schmp. $215^{\circ} \mathrm{C}$ (Lit. ${ }^{[32]} 208^{\circ} \mathrm{C}$ ).

Tab. 5. Silylierung substituierter Halogenpyridine 1, 8

[a] Gemisch aus 1 $\mathbf{e}^{\prime}$ und 2-Brom-5-cyanpyridin [80:20, mit GC (ungeeicht) bestimmt]. - ${ }^{[\mathrm{b}]}$ HMPT und Zugabe von $7.5 \mathrm{~g}(40 \mathrm{mmol})$ 1,2-Dibromethan. 
c) $1 \mathrm{e}^{\prime}:$ Nach Lit. ${ }^{[32]}$, aus $15 \mathrm{~g}(95 \mathrm{mmol})$ 6-Chlornicotinamid, $37.5 \mathrm{ml}(0.41 \mathrm{~mol})$ Phosphoroxychlorid und $125 \mathrm{ml}$ Chloroform, Ausb. $12.5 \mathrm{~g}(94 \%) 1 \mathrm{e}^{\prime}$, Schmp. $118-119^{\circ} \mathrm{C}$ (Ethanol) (Lit. ${ }^{[32]}$ $117-119^{\circ} \mathrm{C}$ ).

2-Brom-5-cyanpyridin: $1.40 \mathrm{~g}(10.0 \mathrm{mmol}) 1 \mathrm{e}^{\prime}$ und $13.5 \mathrm{~g}(50.0$ mmol) Phosphortribromid in $10 \mathrm{ml}$ Chloroform werden $2.5 \mathrm{~d}$ auf $80^{\circ} \mathrm{C}$ erhitzt. Nach Entfernen des Lösungsmittels im Rotationsverdampfer wird der Rückstand auf Eis gegossen, der Niederschlag abfiltriert und für die Elementaranalyse mittels fraktionierter Sublimation gereinigt, Ausb. $1.5 \mathrm{~g}$, Gemisch aus $1 \mathrm{e}^{\prime}$ und 2-Brom-5cyanpyridin [80:20, mit GC (ungeeicht) bestimmt], Schmp. $142^{\circ} \mathrm{C}$.

$\mathrm{C}_{6} \mathrm{H}_{3} \mathrm{BrN}_{2}$ (183.0)

Ber. C 39.38 H 1.65 Br 43.66 N 15.31

Gef. C 40.45 H $1.71 \mathrm{Br} 44.52$ N 15.81

5-(Phenylsulfonyl)-2-(trimethylsilyl) pyridin (7f) nach Lit. ${ }^{[17 a, 33,34]}$

a) 2-Amino-5-iodpyridin: Nach Lit. ${ }^{[33]}$, aus $100 \mathrm{~g}$ (1.06 mol) 2-

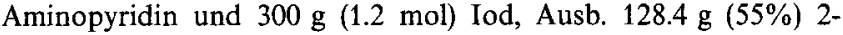
Amino-5-iodpyridin, Schmp. $128-129^{\circ} \mathrm{C}$ (Lit. ${ }^{[3]} 126-128^{\circ} \mathrm{C}$ ).

Tab. 6. Physikalische und ${ }^{1} \mathrm{H}-\mathrm{NMR}-\mathrm{Daten}\left(\mathrm{CDCl}_{3}\right.$, TMS als int. Standard, ס) der substituierten 2-(Trimethylsilyl)pyridine 2, 7 und 9

\begin{tabular}{|c|c|c|c|c|c|c|c|}
\hline & $\begin{array}{l}\text { untaranalyse } \\
\text { (Molmasmenter }\end{array}$ & $\begin{array}{l}\text { Ber. } \\
\text { Gef. }\end{array}$ & C & $\mathrm{H}$ & $\mathrm{N}$ & $\mathrm{Cl}$ & $S$ \\
\hline $2 b$ & $\begin{array}{l}\mathrm{C}_{9} \mathrm{H}_{15} \mathrm{NSi} \\
(165.3)\end{array}$ & & $\begin{array}{l}65.39 \\
65.28\end{array}$ & $\begin{array}{l}9.15 \\
8.95\end{array}$ & $\begin{array}{l}8.47 \\
8.32\end{array}$ & & \\
\hline $2 c$ & $\begin{array}{l}\mathrm{C}_{9} \mathrm{H}_{15} \mathrm{NOSi} \\
(181.3)\end{array}$ & & $\begin{array}{l}59.62 \\
59.45\end{array}$ & $\begin{array}{l}8.34 \\
8.33\end{array}$ & $\begin{array}{l}7.73 \\
7.73\end{array}$ & & \\
\hline $2 d$ & $\begin{array}{l}\mathrm{C}_{8} \mathrm{H}_{12} \mathrm{CINSi} \\
(185.7)\end{array}$ & & $\begin{array}{l}51.74 \\
51.63\end{array}$ & $\begin{array}{l}6.51 \\
6.66\end{array}$ & $\begin{array}{l}7.54 \\
7.67\end{array}$ & $\begin{array}{l}19.09 \\
19.05\end{array}$ & \\
\hline $2 e$ & $\begin{array}{l}\mathrm{C}_{9} \mathrm{H}_{12} \mathrm{~N}_{2} \mathrm{Si} \\
(176.3)\end{array}$ & & $\begin{array}{l}61.32 \\
61.23\end{array}$ & $\begin{array}{l}6.86 \\
6.89\end{array}$ & $\begin{array}{l}15.89 \\
15.76\end{array}$ & & \\
\hline $2 \mathrm{~g}$ & $\begin{array}{l}\mathrm{C}_{11} \mathrm{H}_{21} \mathrm{NSi}_{2} \\
(223.5)\end{array}$ & & $\begin{array}{l}59.12 \\
58.87\end{array}$ & $\begin{array}{l}9.47 \\
9.18\end{array}$ & $\begin{array}{l}6.27 \\
6.30\end{array}$ & & \\
\hline $7 \mathbf{b}$ & $\begin{array}{l}\mathrm{C}_{9} \mathrm{H}_{15} \mathrm{NSi} \\
(165.3)\end{array}$ & & $\begin{array}{l}65.39 \\
65.35\end{array}$ & $\begin{array}{l}9.15 \\
9.11\end{array}$ & $\begin{array}{l}8.47 \\
8.75\end{array}$ & & \\
\hline $7 c$ & $\begin{array}{l}\mathrm{C}_{9} \mathrm{H}_{15} \mathrm{NOSi} \\
(181.3)\end{array}$ & & $\begin{array}{l}59.62 \\
59.87\end{array}$ & $\begin{array}{l}8.34 \\
8.10\end{array}$ & $\begin{array}{l}7.73 \\
8.01\end{array}$ & & \\
\hline $7 d$ & $\begin{array}{l}\mathrm{C}_{8} \mathrm{H}_{12} \mathrm{CINSi} \\
(185.7)\end{array}$ & & $\begin{array}{l}51.74 \\
51.73\end{array}$ & $\begin{array}{l}6.51 \\
6.52\end{array}$ & $\begin{array}{l}7.54 \\
7.64\end{array}$ & $\begin{array}{l}19.09 \\
18.81\end{array}$ & \\
\hline $7 e$ & $\begin{array}{l}\mathrm{C}_{9} \mathrm{H}_{12} \mathrm{~N}_{2} \mathrm{Si} \\
(176.3)\end{array}$ & & $\begin{array}{l}61.32 \\
61.39\end{array}$ & $\begin{array}{l}6.86 \\
6.98\end{array}$ & $\begin{array}{l}15.89 \\
15.76\end{array}$ & & \\
\hline 9 & $\begin{array}{l}\mathrm{C}_{14} \mathrm{H}_{17} \mathrm{NSSi} \\
(259.4)\end{array}$ & & $\begin{array}{l}64.81 \\
64.80\end{array}$ & $\begin{array}{l}6.60 \\
6.60\end{array}$ & $\begin{array}{l}5.40 \\
5.18\end{array}$ & & $\begin{array}{l}12.36 \\
12.09\end{array}$ \\
\hline
\end{tabular}

2b 7.3-7.4 (m, $1 \mathrm{H}, 3-\mathrm{H}), 6.9-7.1(\mathrm{~m}, 1 \mathrm{H}, 5-\mathrm{H}), 8.6-8.8(\mathrm{~m}, 1 \mathrm{H}, 6-\mathrm{H})$, $2.28\left(\mathrm{~s}, 3 \mathrm{H}, \mathrm{CH}_{3}\right)$

2c 7.1-7.2(m, $1 \mathrm{H}, 3-\mathrm{H}), 6.7-7.0(\mathrm{~m}, 1 \mathrm{H}, 5-\mathrm{H}), 8.7-8.8(\mathrm{~m}, 1 \mathrm{H}, 6-\mathrm{H})$, $3.87\left(\mathrm{~s}, 3 \mathrm{H}, \mathrm{OCH}_{3}\right)$

2d 7.5-8.0 (m, $1 \mathrm{H}, 3-\mathrm{H}), 7.1-7.4(\mathrm{~m}, 1 \mathrm{H}, 5-\mathrm{H}), 8.6-8.8(\mathrm{~m}, 1 \mathrm{H}, 6-\mathrm{H})$

2e 7.6-7.7 (m, $1 \mathrm{H}, 3-\mathrm{H}), 7.35-7.5(\mathrm{~m}, 1 \mathrm{H}, 5-\mathrm{H}), 8.92(\mathrm{~d}, J=5.5 \mathrm{~Hz}, 1 \mathrm{H}$, 6-H)

$2 g$ 7.6-7.7 (m, $1 \mathrm{H}, 3-\mathrm{H}), 7.3-7.4(\mathrm{~m}, 1 \mathrm{H}, 5-\mathrm{H}), 8.6-8.8(\mathrm{~m}, 1 \mathrm{H}, 6-\mathrm{H})$, $0.28\left(\mathrm{~s}, 9 \mathrm{H}, \mathrm{Si}\left(\mathrm{CH}_{3}\right)_{3}\right)$

7b $7.3-7.5(\mathrm{~m}, 2 \mathrm{H}, 3-, 4-\mathrm{H}), 8.64(\mathrm{~s}, 1 \mathrm{H}, 6-\mathrm{H}), 2.31\left(\mathrm{~s}, 3 \mathrm{H}, \mathrm{CH}_{3}\right)$

7c 7.34 (dd, $\left.J_{1}=8.2 \mathrm{~Hz}, J_{2}=0.6 \mathrm{~Hz}, 1 \mathrm{H}, 3-\mathrm{H}\right), 7.09\left(\mathrm{dd}, \mathrm{J}_{1}=8.3 \mathrm{~Hz}, \mathrm{~J}_{2}=2.9 \mathrm{~Hz}\right.$, $1 \mathrm{H}, 4-\mathrm{H}), 8.52\left(\mathrm{dd}, J_{1}=2.9 \mathrm{~Hz}, \mathrm{~J}_{2}=0.5 \mathrm{~Hz}, 1 \mathrm{H}, 6-\mathrm{H}\right), 3.84\left(\mathrm{~s}, 3 \mathrm{H}, \mathrm{OCH}_{3}\right)$ 7d 7.5-7.7 (m, $2 \mathrm{H}, 3-, 4-\mathrm{H}), 8.77(\mathrm{~s}, 1 \mathrm{H}, 6-\mathrm{H})$

7e 7.62 (dd, $\left.J_{1}=7.8 \mathrm{~Hz}, J_{2}=1.0 \mathrm{~Hz}, 1 \mathrm{H}, 3-\mathrm{H}\right), 7.84\left(\mathrm{dd}, J_{1}=7.9 \mathrm{~Hz}, J_{2}=2.1 \mathrm{~Hz}\right.$, $1 \mathrm{H}, 4-\mathrm{H}$ ), 8.99 (dd, $\mathrm{J}_{1}=2.0 \mathrm{~Hz}, \mathrm{~J}_{2}=1.0 \mathrm{~Hz}, 1 \mathrm{H}, 6-\mathrm{H}$ )

$97.2-7.4\left(\mathrm{~m}, 6 \mathrm{H}, 3-\mathrm{H}\right.$, Phenyl), $8.47\left(\mathrm{dd}, \mathrm{J}_{1}=7.8 \mathrm{~Hz}, \mathrm{~J}_{2}=2.3 \mathrm{~Hz}, 1 \mathrm{H}, 4-\mathrm{H}\right)$, $8.67\left(\mathrm{dd}, \mathrm{J}_{1}=2.2 \mathrm{~Hz}, \mathrm{~J}_{2}=0.9 \mathrm{~Hz}, 1 \mathrm{H}, 6-\mathrm{H}\right)$

Bei allen Verbindungen: 0.30-0.37 [s, $\left.9 \mathrm{H}, \mathrm{Si}\left(\mathrm{CH}_{3}\right)_{3}\right]$ b) 2-Amino-5-(phenylthio) pyridin: Nach Lit. ${ }^{[34]}$, aus $10.0 \mathrm{~g} \mathrm{(45}$ $\mathrm{mmol}$ ) 2-Amino-5-iodpyridin, $1.47 \mathrm{~g} \mathrm{(64} \mathrm{mmol)} \mathrm{Natrium,} 7.1 \mathrm{~g}(64$ mmol) Thiophenol, $0.9 \mathrm{~g}$ Kupferpulver und $80 \mathrm{ml}$ Ethanol, Ausb. $6.0 \mathrm{~g}(66 \%)$ 2-Amino-5-(phenylthio)pyridin, Schmp. $122-124^{\circ} \mathrm{C}$ (Lit. ${ }^{[34]} 123-125^{\circ} \mathrm{C}$ ).

c) 2-Brom-5-(phenylthio ) pyridin (8): Nach Lit. ${ }^{[17 a]}$, aus $63.4 \mathrm{~g}(0.31$ mol) 2-Amino-5-(phenylthio)pyridin, $110.0 \mathrm{~g}(0.69 \mathrm{~mol})$ Brom, $44.0 \mathrm{~g}(0.64 \mathrm{~mol}) \mathrm{NaNO}_{2}$ in $600 \mathrm{ml}$ Wasser und $200 \mathrm{ml}(1.7 \mathrm{~mol})$ 48proz. $\mathrm{HBr}$, Ausb. $25.1 \mathrm{~g} \mathrm{(30 \% )} \mathrm{8,} \mathrm{Sdp.} 98-100^{\circ} \mathrm{C} / 0.005$ Torr.

d) 7f: Man gibt zu $4.40 \mathrm{~g}$ (17.0 mmol) 9 in $40 \mathrm{ml}$ Dichlormethan bei $-70^{\circ} \mathrm{C}$ innerhalb $1 \mathrm{~h} 7.13 \mathrm{~g}(41.0 \mathrm{mmol}) \mathrm{m}$-Chlorperbenzoesäure in $40 \mathrm{ml}$ Dichlormethan. Nach $2 \mathrm{~h}$ bei $-10^{\circ} \mathrm{C}$ wird $m$-Chlorbenzoesäure abfiltriert und das Filtrat $3 \mathrm{~h}$ mit festem Kaliumcarbonat gerührt. Nach Abfiltrieren wird das Filtrat im Rotationsverdampfer eingeengt und der Rückstand über Aluminiumoxid mit Petrolether/Ethylacetat (5:1) chromatographiert, Ausb. $1.25 \mathrm{~g}$ (26\%) 7f, Schmp. $112-115^{\circ} \mathrm{C} .-{ }^{1} \mathrm{H}-\mathrm{NMR}\left(\mathrm{CDCl}_{3}\right): \delta=0.34[\mathrm{~s}$, $\left.9 \mathrm{H}, \mathrm{Si}\left(\mathrm{CH}_{3}\right)_{3}\right], 7.5-7.6(\mathrm{~m}, 3 \mathrm{H}, m, p-\mathrm{H} \mathrm{Ph}), 7.66\left(\mathrm{dd}, J_{1}=7.85, J_{2}\right.$ $=0.93 \mathrm{~Hz}, 1 \mathrm{H}, 3-\mathrm{H}), 8.0-8.1(\mathrm{~m}, 2 \mathrm{H}, o-\mathrm{H} \mathrm{Ph}), 8.11\left(\mathrm{dd}, J_{1}=8.03\right.$, $\left.J_{2}=2.32 \mathrm{~Hz}, 1 \mathrm{H}, 4-\mathrm{H}\right), 9.25\left(\mathrm{dd}, J_{1}=2.28, J_{2}=0.91 \mathrm{~Hz}, 1 \mathrm{H}, 6-\right.$ H).

$$
\begin{aligned}
& \mathrm{C}_{14} \mathrm{H}_{17} \mathrm{NO}_{2} \mathrm{SSi}(291.4) \\
& \text { Ber. C } 57.70 \text { H } 5.88 \mathrm{~N} 4.81 \mathrm{~S} 11.01 \\
& \text { Gef. C } 57.60 \mathrm{H} 5.90 \mathrm{~N} 4.64 \mathrm{~S} 10.90
\end{aligned}
$$

Silylierung von 4- bzw. 5-substituierten Halogenpyridinen 1 und 8. Allgemeines: Unter Stickstoff wird Magnesium unter Erwärmung mit etwas Iod angeätzt. Nach Entfernen von überschüssigem Iod i. Vak. wird jeweils $1 / 4$ von Tetrahydrofuran (THF) und Chlortrimethylsilan (TMCS) zugegeben und auf $70^{\circ} \mathrm{C}$ erhitzt $\left[90^{\circ} \mathrm{C}\right.$ bei

\begin{tabular}{|c|c|c|c|c|c|c|}
\hline \multicolumn{2}{|c|}{$\begin{array}{l}\text { Edukte } \\
\mathrm{g} \text { (mmol) }\end{array}$} & \multirow{2}{*}{$\begin{array}{l}\begin{array}{c}\text { Benzaldehyd } \\
\mathrm{g} \text { (mmol) }\end{array} \\
2.33 \\
(22.0)\end{array}$} & \multirow{2}{*}{$\begin{array}{l}\text { Reaktions- } \\
\text { zeit [h] }\end{array}$} & \multicolumn{2}{|c|}{$\begin{array}{r}\text { Produkte } \\
\text { Ausb. } \\
\mathrm{g}(\%)\end{array}$} & \multirow{2}{*}{$\begin{array}{l}\begin{array}{l}\text { Sdp. } \\
{\left[{ }^{\circ} \mathrm{C} / \text { Torr }\right]}\end{array} \\
76-79 / 0.01\end{array}$} \\
\hline $2 a^{a)}$ & $\begin{array}{l}3.03 \\
(20.0)\end{array}$ & & & $\mathbf{3 a}$ & $\begin{array}{l}4.58 \\
(89)\end{array}$ & \\
\hline $2 b$ & $\begin{array}{l}3.31 \\
(20.0)\end{array}$ & $\begin{array}{l}2.33 \\
(22.0)\end{array}$ & 2.7 & $\mathbf{3 b}$ & $\begin{array}{l}4.36 \\
(81)\end{array}$ & $88-90 / 0.01$ \\
\hline $2 c$ & $\begin{array}{l}1.81 \\
(10.0)\end{array}$ & $\begin{array}{l}9.17 \\
(11.0)\end{array}$ & 2.5 & $3 c$ & $\begin{array}{l}2.18 \\
(76)\end{array}$ & $\begin{array}{r}110-115 / \\
0.005\end{array}$ \\
\hline $2 d$ & $\begin{array}{l}0.19 \\
(1.0)\end{array}$ & $\begin{array}{l}0.20 \\
(1.9)\end{array}$ & 2.0 & $3 d$ & $\begin{array}{l}0.24 \\
(82)\end{array}$ & $110 / 0.01$ \\
\hline $2 e$ & $\begin{array}{l}0.88 \\
(5.0)\end{array}$ & $\begin{array}{l}0.85 \\
(8.0)\end{array}$ & 3 & $3 e$ & $\begin{array}{l}0.70 \\
(50)\end{array}$ & $122 / 0.01$ \\
\hline $2 f$ & $\begin{array}{l}0.75 \\
(2.57)\end{array}$ & $\begin{array}{l}0.53 \\
(5.0)\end{array}$ & 4 & $3 t$ & $\begin{array}{l}0.39 \\
(39)\end{array}$ & - \\
\hline $7 b$ & $\begin{array}{l}0.83 \\
(5.0)\end{array}$ & $\begin{array}{l}0.85 \\
(8.0)\end{array}$ & 3 & 3b' & $\begin{array}{l}1.01 \\
(75)\end{array}$ & $86 / 0.001$ \\
\hline $7 c$ & $\begin{array}{l}0.48 \\
(2.64)\end{array}$ & $\begin{array}{l}1.0 \\
(9.43)\end{array}$ & 16 & $3 c^{\prime}$ & $\begin{array}{l}0.52 \\
(69)\end{array}$ & $98 / 0.002$ \\
\hline $7 d$ & $\begin{array}{l}1.0 \\
(5.4)\end{array}$ & $\begin{array}{l}0.85 \\
(8.0)\end{array}$ & 3 & $3 d^{\prime}$ & $\begin{array}{l}0.80 \\
(51)\end{array}$ & $88 / 0.01$ \\
\hline $7 e$ & $\begin{array}{l}0.26 \\
(1.5)\end{array}$ & $\begin{array}{l}0.80 \\
(7.5)\end{array}$ & 16 & $3 e^{\prime}$ & $\begin{array}{l}0.20 \\
(47)\end{array}$ & $105 / 0.0001$ \\
\hline $7 f$ & $\begin{array}{l}0.16 \\
(0.55)\end{array}$ & $\begin{array}{l}0.29 \\
(2.73)\end{array}$ & 12 & $3 f^{\prime}$ & $\begin{array}{l}0.09 \\
(41)\end{array}$ & - \\
\hline $5 a^{a)}$ & $\begin{array}{l}3.02 \\
(20.0)\end{array}$ & $\begin{array}{l}2.12 \\
(20.0)\end{array}$ & $34^{\text {b) }}$ & $6 a$ & $\begin{array}{l}2.51 \\
(49)\end{array}$ & $84 / 0.01$ \\
\hline $5 b^{a)}$ & $\begin{array}{l}3.02 \\
(20.0)\end{array}$ & $\begin{array}{l}2.12 \\
(20.0)\end{array}$ & $2^{\text {c) }}$ & $6 b$ & $\begin{array}{l}3.79 \\
(74)\end{array}$ & $105 / 0.02$ \\
\hline
\end{tabular}

Tab. 7. Umsetzung der substituierten (Trimethylsilyl)pyridine 2, 7 und 5 mit Benzaldehyd

[a] Lit. ${ }^{[10 a]}$ - ${ }^{[b]}$ Mit $0.61 \mathrm{~g}(4.0 \mathrm{mmol})$ Caesiumfluorid, $90^{\circ} \mathrm{C}$. ${ }^{[c]}$ Mit $0.30 \mathrm{~g}(2.0 \mathrm{mmol})$ Caesiumfluorid, $90^{\circ} \mathrm{C}$. 
Hexamethylphosphorsäuretriamid (HMPT)]. Das verbleibende THF und TMCS wird mit 1 bzw. 8 vermischt. 5-10\% dieses Eduktgemisches werden schnell zugetropft, und nach Starten der Grignard-Reaktion wird die restliche Lösung unter Rühren innerhalb 20 min so zugetropft, daß die Reaktionslösung schwach siedet. Es wird $1 \mathrm{~h}$ unter Rückfluß erhitzt, dann das abgekühlte Reaktionsgemisch auf kalte, ges. Natriumhydrogencarbonat-Lösung gegossen und die Mischung mit Diethylether extrahiert. Die vereinigten Etherextrakte werden mit Wasser gewaschen, mit Natriumsulfat getrocknet und im Rotationsverdampfer i.Vak. eingeengt. Der

Tab. 8. Elementaranalytische und ${ }^{1} \mathrm{H}-\mathrm{NMR}$-Daten $\left(\mathrm{CDCl}_{3}\right.$, TMS als int. Standard, $\delta$ ) der substituierten [ $\alpha$-(Trimethylsiloxy)benzyl]pyridine 3 und 6

\begin{tabular}{|c|c|c|c|c|c|c|c|}
\hline $\begin{array}{r}\text { Elem } \\
\text { Su } \\
\text { (I }\end{array}$ & $\begin{array}{l}\text { Ientaranalyse } \\
\text { mmenformel } \\
\text { Molmasse) }\end{array}$ & $\begin{array}{l}\text { Ber. } \\
\text { Gef. }\end{array}$ & C & $\mathrm{H}$ & $\mathbf{N}$ & $\mathrm{Cl}$ & $\mathrm{S}$ \\
\hline $3 \mathbf{a}$ & $\begin{array}{l}\mathrm{C}_{15} \mathrm{H}_{19} \mathrm{NOSi} \\
(257.4)\end{array}$ & & $\begin{array}{l}69.99 \\
70.24\end{array}$ & $\begin{array}{l}7.44 \\
7.35\end{array}$ & $\begin{array}{l}5.44 \\
5.19\end{array}$ & & \\
\hline $3 b$ & $\begin{array}{l}\mathrm{C}_{16} \mathrm{H}_{21} \mathrm{NOSi} \\
(271,4)\end{array}$ & & $\begin{array}{l}70.80 \\
70.84\end{array}$ & $\begin{array}{l}7.80 \\
7.93\end{array}$ & $\begin{array}{l}5.16 \\
5.33\end{array}$ & & \\
\hline $3 c$ & $\begin{array}{l}\mathrm{C}_{16} \mathrm{H}_{21} \mathrm{NO}_{2} \mathrm{Si} \\
(287.4)\end{array}$ & & $\begin{array}{l}66.86 \\
67.08\end{array}$ & $\begin{array}{l}7.36 \\
7.40\end{array}$ & $\begin{array}{l}4.87 \\
5.01\end{array}$ & & \\
\hline $3 d$ & $\begin{array}{l}\mathrm{C}_{15} \mathrm{H}_{18} \mathrm{CINOSi} \\
(291.9)\end{array}$ & & $\begin{array}{l}61.73 \\
62.04\end{array}$ & $\begin{array}{l}6.22 \\
6.34\end{array}$ & $\begin{array}{l}4.80 \\
5.04\end{array}$ & $\begin{array}{l}12.15 \\
12.04\end{array}$ & \\
\hline $3 e$ & $\begin{array}{l}\mathrm{C}_{16} \mathrm{H}_{18} \mathrm{~N}_{2} \mathrm{OSi} \\
(282.4)\end{array}$ & & $\begin{array}{l}68.05 \\
67.85\end{array}$ & $\begin{array}{l}6.42 \\
6.46\end{array}$ & $\begin{array}{l}9.92 \\
9.71\end{array}$ & & \\
\hline $3 f$ & $\begin{array}{l}\mathrm{C}_{21} \mathrm{H}_{23} \mathrm{NO}_{3} \mathrm{SSi} \\
(397.6)\end{array}$ & & $\begin{array}{l}63.44 \\
63.55\end{array}$ & $\begin{array}{l}5.83 \\
5.82\end{array}$ & $\begin{array}{l}3.52 \\
3.50\end{array}$ & & $\begin{array}{l}8.07 \\
8.03\end{array}$ \\
\hline 3b' & $\begin{array}{l}\mathrm{C}_{16} \mathrm{H}_{21} \mathrm{NOSi} \\
(271.4)\end{array}$ & & $\begin{array}{l}70.80 \\
70.64\end{array}$ & $\begin{array}{l}7.80 \\
7.65\end{array}$ & $\begin{array}{l}5.16 \\
5.07\end{array}$ & & \\
\hline $3 c^{\prime}$ & $\begin{array}{l}\mathrm{C}_{16} \mathrm{H}_{21} \mathrm{NO}_{2} \mathrm{Si} \\
(287.4)\end{array}$ & & $\begin{array}{l}66.86 \\
66.93\end{array}$ & $\begin{array}{l}7.36 \\
7.22\end{array}$ & $\begin{array}{l}4.87 \\
4.84\end{array}$ & & \\
\hline $3 d^{\prime}$ & $\begin{array}{l}\mathrm{C}_{15} \mathrm{H}_{18} \mathrm{CINOSi} \\
(291.9)\end{array}$ & & $\begin{array}{l}61.73 \\
61.97\end{array}$ & $\begin{array}{l}6.22 \\
5.93\end{array}$ & $\begin{array}{l}4.80 \\
4.89\end{array}$ & $\begin{array}{l}12.15 \\
11.91\end{array}$ & \\
\hline $3 e^{\prime}$ & $\begin{array}{l}\mathrm{C}_{16} \mathrm{H}_{18} \mathrm{~N}_{2} \mathrm{OSi} \\
(282.4)\end{array}$ & & $\begin{array}{l}68.05 \\
67.80\end{array}$ & $\begin{array}{l}6.42 \\
6.38\end{array}$ & $\begin{array}{l}9.92 \\
9.88\end{array}$ & & \\
\hline $31^{\prime}$ & $\begin{array}{l}\mathrm{C}_{21} \mathrm{H}_{23} \mathrm{NO}_{3} \mathrm{SSi} \\
(397.6)\end{array}$ & & $\begin{array}{l}63.44 \\
63.60\end{array}$ & $\begin{array}{l}5.83 \\
5.76\end{array}$ & $\begin{array}{l}3.52 \\
3.72\end{array}$ & & $\begin{array}{l}8.07 \\
8.21\end{array}$ \\
\hline $6 a$ & $\begin{array}{l}\mathrm{C}_{15} \mathrm{H}_{19} \text { NOSi } \\
(257.4)\end{array}$ & & $\begin{array}{l}69.99 \\
69.72\end{array}$ & $\begin{array}{l}7.44 \\
7.33\end{array}$ & $\begin{array}{l}5.44 \\
5.41\end{array}$ & & \\
\hline $6 b$ & $\begin{array}{l}\mathrm{C}_{15} \mathrm{H}_{19} \mathrm{NOSi} \\
(257.4)\end{array}$ & & $\begin{array}{l}69.99 \\
70.09\end{array}$ & $\begin{array}{l}7.44 \\
7.33\end{array}$ & $\begin{array}{l}5.44 \\
5.48\end{array}$ & & \\
\hline
\end{tabular}

${ }^{1} \mathrm{H}-\mathrm{NMR}$

3a $\quad 6.08(\mathrm{~s}, 1 \mathrm{H}, \mathrm{CH}), 7.2-7.9(\mathrm{~m}, 8 \mathrm{H}, 3-, 4-, 5-\mathrm{H}$, Phenyl), 8.6-8.8 (m, $1 \mathrm{H}, 6-\mathrm{H})$

3b $5.93(\mathrm{~s}, 1 \mathrm{H}, \mathrm{CH}), 7.2-7.6(\mathrm{~m}, 6 \mathrm{H}, 3-\mathrm{H}$, Phenyl), 6.9-7.1 (m, $1 \mathrm{H}, 5-\mathrm{H}), 8.3-$ $8.5(\mathrm{~m}, 1 \mathrm{H}, 6-\mathrm{H}), 2.32\left(\mathrm{~s}, 3 \mathrm{H}, \mathrm{CH}_{3}\right)$

3c $\quad 5.85(\mathrm{~s}, 1 \mathrm{H}, \mathrm{CH}), 7.1-7.6(\mathrm{~m}, 6 \mathrm{H}, 3-\mathrm{H}$, Phenyl), 6.5-6.7 (m, $1 \mathrm{H}, 5-\mathrm{H}), 8.3-$ $8.5(\mathrm{~m}, 1 \mathrm{H}, 6-\mathrm{H}), 3.80\left(\mathrm{~s}, 3 \mathrm{H}, \mathrm{OCH}_{3}\right)$

3d $5.80(\mathrm{~s}, 1 \mathrm{H}, \mathrm{CH}), 6.9-7.5(\mathrm{~m}, 6 \mathrm{H}, 3-\mathrm{H}$, Phenyl), 7.5-7.6 (m, $1 \mathrm{H}, 5-\mathrm{H}), 8.2$ $8.4(\mathrm{~m}, 1 \mathrm{H}, 6-\mathrm{H})$

3e $5.95(\mathrm{~s}, 1 \mathrm{H}, \mathrm{CH}), 7.93(\mathrm{~s}, 1 \mathrm{H}, 3-\mathrm{H}), 7.2-7.6(\mathrm{~m}, 6 \mathrm{H}, 5-\mathrm{H}$, Phenyl), 8.6 (d, $J=5.0 \mathrm{~Hz}, 1 \mathrm{H}, 6-\mathrm{H})$

3f $5.88(\mathrm{~s}, 1 \mathrm{H}, \mathrm{CH}), 8.09(\mathrm{~s}, 1 \mathrm{H}, 3-\mathrm{H}), 7.3-7.65(\mathrm{~m}, 11 \mathrm{H}, 5-\mathrm{H}$, Phenyl,H-Ph$\mathrm{SO}_{2}$ ), 8.64 (dd, $J_{1}=5.1 \mathrm{~Hz}, J_{2}=0.7 \mathrm{~Hz}, 1 \mathrm{H}, 6-\mathrm{H}$ )

3b' $5.86(\mathrm{~s}, 1 \mathrm{H}, \mathrm{CH}), 7.4-7.45(\mathrm{~m}, 7 \mathrm{H}, 3-, 4-\mathrm{H}$, Phenyl), $8.3(\mathrm{~s}, 1 \mathrm{H}, 6-\mathrm{H}), 2.26$ (s, $\left.3 \mathrm{H}_{1} \mathrm{CH}_{3}\right)$

$3 c^{\prime} \quad 5.86(\mathrm{~s}, 1 \mathrm{H}, \mathrm{CH}), 7.1-7.5\left(\mathrm{~m}, 7 \mathrm{H}, 3-, 4-\mathrm{H}\right.$, Phenyl), $8.2\left(\mathrm{dd}, \mathrm{J}_{1}=2.9 \mathrm{~Hz}\right.$, $\left.J_{2}=0.4 \mathrm{~Hz}, 1 \mathrm{H}, 6-\mathrm{H}\right), 3.80\left(\mathrm{~s}, 3 \mathrm{H}, \mathrm{OCH}_{3}\right)$

3d' $5.96(\mathrm{~s}, 1 \mathrm{H}, \mathrm{CH}), 7.64(\mathrm{~d}, J=8.5 \mathrm{~Hz}, 1 \mathrm{H}, 3-\mathrm{H}), 7.72\left(\mathrm{dd}, J_{1}=8.5 \mathrm{~Hz}\right.$, $\left.J_{2}=2.4 \mathrm{~Hz}, 1 \mathrm{H}, 4-\mathrm{H}\right), 8.54(\mathrm{~d}, J=2.1 \mathrm{~Hz}, 1 \mathrm{H}, 6-\mathrm{H}), 7.3-7.5(\mathrm{~m}, 5 \mathrm{H}$, Phenyl)

3e' $5.95(\mathrm{~s}, 1 \mathrm{H}, \mathrm{CH}), 7.25-8.25(\mathrm{~m}, 7 \mathrm{H}, 3-4-\mathrm{H}$, Phenyl), 8.8-8.9 (m, $1 \mathrm{H}, 6-\mathrm{H})$

$37 \quad 5.87(\mathrm{~s}, 1 \mathrm{H}, \mathrm{CH}), 7.76(\mathrm{~d}, J=8.4 \mathrm{~Hz}, 1 \mathrm{H}, 3-\mathrm{H}), 8.16\left(\mathrm{dd}, J_{1}=8.3 \mathrm{~Hz}\right.$, $\left.J_{2}=2.5 \mathrm{~Hz}, 1 \mathrm{H}, 4-\mathrm{H}\right), 8.97\left(\mathrm{dd}, J_{1}=2.3 \mathrm{~Hz}, J_{2}=0.6 \mathrm{~Hz}, 1 \mathrm{H}, 6-\mathrm{H}\right), 7.2-7.6(\mathrm{~m}$, $8 \mathrm{H}$, Phenyl, $\left.m-, \mathrm{p}-\mathrm{H}-\mathrm{PhSO}_{2}\right), 7.9-8.0\left(\mathrm{~m}, 2 \mathrm{H}, \mathrm{O}-\mathrm{H}-\mathrm{PhSO}_{2}\right)$

6a $\quad 5.83(\mathrm{~s}, 1 \mathrm{H}, \mathrm{CH}), 7.6-7.8(\mathrm{~m}, 1 \mathrm{H}, 4-\mathrm{H}), 7.1-7.4(\mathrm{~m}, 1 \mathrm{H}, 5-\mathrm{H}), 8.3-8.7(\mathrm{~m}$ $2 \mathrm{H}, 2-, 6-\mathrm{H}), 7.36(\mathrm{~m}, 5 \mathrm{H}$, Phenyl)

6b $\quad 5.79(\mathrm{~s}, 1 \mathrm{H}, \mathrm{CH}), 7.3-7.45(\mathrm{~m}, 7 \mathrm{H}, 3-, 5-\mathrm{H}$, Phenyl), 8.5-8.6 (m, $2 \mathrm{H}, 2-, 6-\mathrm{H})$

Bei allen Verbindungen im Bereich 0.05-0.14 $\left[\mathrm{s}, 9 \mathrm{H}, \mathrm{Si}\left(\mathrm{CH}_{3}\right)_{3}\right]$
Rückstand wird destilliert oder über Kieselgel mit Petrolether/ Ethylacetat (2:1 oder 5:1) chromatographiert.

Umsetzung der substituierten 2-(Trimethylsilyl)pyridine 2 und 7 mit Benzaldehyd. Allgemeines: Unter Stickstoff werden 2 oder 7 und Benzaldehyd in einen mit Stickstoff gespülten Kolben gegeben und die jeweils angegebene Zeit auf $100^{\circ} \mathrm{C}$ erhitzt. Nach Entfernen von überschüssigem Benzaldehyd werden die Verbindungen 3 i. Hochvak. destilliert.

Kinetikversuche. Allgemeines: In einen ausgeheizten und mit Stickstoff gespülten 25-ml-Zweihalskolben werden Benzaldehyd und DMF mittels einer ausgeheizten Plastikspritze gegeben. Es wird mehrfach entgast und mit Stickstoff gesättigt. Nach Erreichen der Reaktionstemp. $\left(55.3^{\circ} \mathrm{C} \pm 0.2\right)$ wird 2 bzw. 7 zugesetzt. Da die Reaktionsverfolgung mittels Gaschromatographie relativ hohe Edukt-Konzentrationen erfordert, wird DMF in 2.7fach molarem Überschuß, bezogen auf Benzaldehyd, eingesetzt. In festen Abständen werden Proben von $0.1 \mathrm{ml}$ entnommen, mit $0.4 \mathrm{ml}$ Diethylether versetzt und bis zur gaschromatographischen Untersuchung bei $-25^{\circ} \mathrm{C}$ aufbewahrt. Als interner Standard wird Diphenylether eingesetzt (Stammlösung in DMF: $100.0 \mathrm{mmol} / \mathrm{kg}$ )

\section{CAS-Registry-Nummern}

1a: 109-09-1 / 1 b: 4926-28-7 / 1 b': 3510-66-5 / 1 c: 89488-29-9 / 1 c': 139585-48-1 / 1d: 22918-01-0 / 1 d': 40473-01-6 / 1e: $33252-$ 30-1 / 1 e': 33252-28-7 / 1 g: 26452-80-2 / 2a: 13737-04-7 / 2b: 19854 23-0 / 2c: $139585-49-2$ /2d: 139585-50-5/2e: $139585-51-6 / 2 f$ : 139585-52-7 / 2g: 139585-53-8 / 3a: 22843-49-8 / 3b: 139585-54-9 3b': 139585-59-4 / 3c: 139585-55-0 / 3c': 139585-69-6 / 3d: 139585

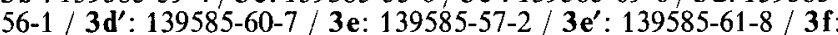
139585-58-3 / 3f': 139585-62-9 / 4a: 626-55-1 / 4b: 1120-87-2 / 5a 17379-37-2 / 5b: 18301-46-7 / 6a: 139585-63-0 / 6b: 58163-76-1 7b: 13737-09-2 / 7c: 139585-64-1/7d: 139585-65-2/7e: 13958566-3 / 7f: 139585-67-4 / 8: 67743-63-9 / 9: 139585-68-5 / PhCHO: 100-52-7 / 2-Amino-4-methylpyridin: 695-34-1 / 2-Amino-5-methylpyridin: 1603-41-4 / 2-Amino-5-chlorpyridin: 1072-98-6 2-Brompyridin: 109-04-6 / 2-Brompyridin- $N$-oxid: 14305-17-0 / 2-Brom-4-nitropyridin- $N$-oxid: 52092-43-0 / 2-Brom-4-nitropyridin: 6945-67-1 / 4-Amino-2-brompyridin: 7598-35-8 / 2-Brom-4 (nitroamino)pyridin: 15367-12-1 / Isonicotinsäure-methylester: 2459-09-8 / Isonicotinamid: 1453-82-3 / Isonicotinamid- $N$-oxid: 38557-82-3 / 4-Nitropyridin- $N$-oxid: $1124-33-0$ / 2-Amino-5-nitropyridin: 4214-76-0 / 5-Nitro-2 $(1 H)$-pyridinon: 5418-51-9 / 2-Chlor5-nitropyridin: 4548-45-2 / 5-Amino-2-chlorpyridin: 5350-93-6 2-Chlor-5-hydroxypyridin: 41288-96-4 / Cumalinsäure-methylester: 6018-41-3 / 6-Hydroxynicotinsäure: 5006-66-6 / 6-Chlornicotinamid: 6271-78-9 / 2-Brom-5-cyanpyridin: 139585-70-9 / 2 Aminopyridin: 504-29-0 / 2-Amino-5-iodpyridin: 20511-12-0 / 2-Amino-5-(phenylthio)pyridin: 64064-25-1

${ }^{[1]}$ Zur elektrophilen Aromatensubstitution, 39. - 38. Mitteilung: F. Effenberger, W. Daub, Chem. Ber. 1991, 124, 2113-2118.

${ }^{[2]}{ }^{[2 a]}$ A. Krebs, Dissertation, Univ. Stuttgart, 1982. - [2b] P. Willrett, Diplomarbeit, Univ. Stuttgart, 1985, und Dissertation, Univ. Stuttgart, 1988.

[3] J. Dunogues, Ann. Chim. (Paris) 1983, 8, 135-149.

[4] [4a] F. Effenberger, W. Spiegler, Chem. Ber. 1985, 118 3872-3899. - [a] J. March, Advanced Organic Chemistry, 3. Aufl., Wiley, New York, 1985, S. $304 \mathrm{ff}$ - ${ }^{14 \mathrm{c}]}$ J. O. Edwards, R. G. Pearson, J. Am. Chem. Soc. 1962, 84, 16-24.

${ }^{[5]}{ }^{[5 a]}$ F. Effenberger, A. Krebs, J. Org. Chem. 1984, 49, 4687-4695. - ${ }^{[5 b]}$ F. Effenberger, M. Roos, R. Ahmad, A. Krebs, Chem. Ber. 1991, 124, 1639-1650.

${ }^{[6]}{ }^{[6 a]}$ F. H. Pinkerton, S. F. Thames, J. Heterocycl. Chem. 1969, 6 , 433. - ${ }^{\left[{ }^{6 b]}\right.}$ F. H. Pinkerton, S. F. Thames, J. Organomet. Chem. 1970, 24, 623-627.

${ }^{[7]}$ L. Birkofer, O. Stuhl, Top. Curr. Chem. 1980, 88, 33-88.

${ }^{[8]}$ P. Jutzi, H. J. Hoffmann, Chem. Ber. 1973, 106, 594-605.

${ }^{[9]}{ }^{[9 a]}$ D. Häbich, Dissertation, Univ. Stuttgart, 1977. - ${ }^{[9 b]}$ F. Ef fenberger, J. König, Tetrahedron 1988, 44, $3281-3288$. 
[10] 110a] F. Effenberger, D. Häbich, Liebigs Ann. Chem. 1979, $842-857$ [ $^{10 \mathrm{~b}]}$ D. Häbich, F. Effenberger, Synthesis 1979, $841-876$.

${ }^{[11]}$ C. H. DePuy, V. M. Bierbaum, L. A. Flippin, J. J. Grabowski, G. K. King, R. J. Schmitt, S. A. Sullivan, J. Am. Chem. Soc. 1980, $102,5012-5015$.

${ }^{[12]}$ J. A. Zoltewicz, G. Grahe, C. L. Smith, J. Am. Chem. Soc. 1969, $91,5501-5505$

[13] [13a] B. Nowak-Wydra, M. Szafran, J. Org. Chem. 1983, 48, $2327-2329 .-{ }^{[13 b]}$ R. S. Brown, H. Slebocka-Tilk, J. M. Buschek, J. G. Ulan, J. Am. Chem. Soc. 1984, 106, 5979-5984.

${ }^{[14]}{ }^{[14 a]}$ D. G. Anderson, M. A. M. Bradney, D. E. Webster, J. Chem. Soc. B 1968, 450-452. - ${ }^{[14 b]}$ P. Jutzi, O. Lorey, J. Organomet Chem. 1976, 104, 153-160. - ${ }^{[14 c]}$ G. Seconi, C. Eaborn, $J$. Chem. Soc., Perkin Trans 2, 1981, 1051-1056. - ${ }^{[14 d]}$ R. J. P. Corriu, C. Guerin, Adv. Organomet. Chem. 1982, 20, 265-312.

[15] [15a] E. Anders, T. Gaßner, Angew. Chem. 1982, 94, 292-293 Angew. Chem. Int. Ed. Engl. 1982, 21, 289. - ${ }^{[156]}$ E. Anders, $\mathrm{H}$ G. Boldt, R. Fuchs, T. Gaßner, Tetrahedron Lett. 1984, 25 , $1715-1716$

[16] J. A. Zoltewicz, L. S. Helmick, J. Am. Chem. Soc. 1970, 92 $7547-7552$

[17] [17a] L. C. Craig, J. Am. Chem. Soc. 1934, 56, 231-232. ${ }^{[17 b]}$ F. H. Case, J. Am. Chem. Soc. 1946, 68, 2574-2577.

${ }^{[18]}$ E. Shaw, J. Bernstein, K. Losee, W. A. Lott, J. Am. Chem. Soc 1950, $72,4362-4364$

${ }^{[19]}$ H. J. den Hertog, C. R. Kolder, W. P. Combé, Rec. Trav. Chim. Pays-Bas 1951, 70, $591-599$.

${ }^{[20]}$ Z. Talik, T. Talik, Rocz. Chem. 1962, 36, 417-423; Chem. Abstr. $1963,58,5627$.

${ }^{[21]}$ Z. Talik, Rocz. Chem. 1962, 36, 1313-1320; Chem. Abstr. 1963, 59,6358 .

${ }^{[22]}$ P. C. Jain, S. K. Chatterjee, N. Anand, Indian J. Chem. 1966, 4, $403-405$

[23] L. W. Deady, O. L. Korytsky, J. E. Rowe, Aust. J. Chem. 1982, $35,2025-2034$.

${ }^{[24]}$ T. Talik, Z. Talik, Rocz. Chem. 1969, 43, 489-497; Chem. Abstr. 1969, 71, $12972 \mathrm{x}$.

${ }^{125]}$ B. Prijs, A. H. Lutz, H. Erlenmeyer, Helv. Chim. Acta 1948, 31, $571-577$

[26] D. Libermann, N. Rist, F. Grumbach, S. Cals, M. Moyeux, A. Rouaix, Bull. Soc. Chim. Fr. 1958, 694-702.

${ }^{[27]}$ T. Itai, Yakugaku Zasshi 1945, 65, 70; Chem. Abstr. 1951, 45, 8525 .

[28] [28a] W. T. Caldwell, E. C. Kornfeld, J. Am. Chem. Soc. 1942, 64, 1695-1698. - ${ }^{[286]}$ M. A. Phillips, J. Chem. Soc. 1941, 9-15.
${ }^{[29]}$ C. O. Okafor, J. Org. Chem. 1973, 38, 4383-4386.

${ }^{[30]}{ }^{[30 a]}$ R. D. Westland, R. A. Cooley, J. L. Holmes, J. S. Hong, M. H. Lin, M. L. Zwiesler, J. Med. Chem. 1973, 16, 319-327. ${ }^{[30 b]}$ P. Nedenskov, N. Clauson-Kaas, J. Lei, H. Heide, G. Olsen, G. Jansen, Acta Chem. Scand. 1969, 23, 1791-1796.

[31] J. H. Boyer, W. Schoen in Organic Syntheses Coll. Vol. IV (Hrsg.: N. Rabjohn), Wiley, New York, 1963, S. 532-534.

${ }^{{ }^{32]} \mathrm{H}}$ H. S. Forrest, J. Walker, J. Chem. Soc. 1948, 1939-1945.

${ }^{\left[{ }^{33]}\right.}$ W. T. Caldwell, F. T. Tyson, L. Lauer, J. Am. Chem. Soc. 1944 $66,1479-1484$.

${ }^{[34]}$ R. J. Bochis, R. A. Dybas, P. Eskola, P. Kulsa, B. O. Linn, A Lusi, E. P. Meitzner, J. Milkowski, H. Mrozik, L. E. Olen, L. H. Peterson, R. L. Tolman, A. F. Wagner, F. S. Waksmunski, J. R. Egerton, D. A. Ostlind, J. Med. Chem. 1978, 21, 235-237.

${ }^{[35]}$ D. E. Pearson, D. Cowan, J. D. Beckler, J. Org. Chem. 1959, 24 $504-509$.

[36] [36a] D. G. Anderson, D. E. Webster, J. Chem. Soc. B 1968 1008-1009. - ${ }^{[36 \mathrm{~b}]}$ R. W. Bott, C. Eaborn, P. M. Jackson, J Organomet. Chem. 1967, 7, 79-83. - ${ }^{[36 c]}$ D. G. Anderson, D. E. Webster, J. Organomet. Chem. 1968, 13, 113-116.

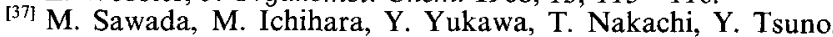
Bull. Chem. Soc. Jpn. 1980, 53, 2055-2060.

[38] [38a] J. M. Abboud, J. Catalan, J. Elguero, R. W. Taft, J. Org Chem. 1988, 53,1137-1140. - ${ }^{[38 b]}$ G. Butt, R. D. Topsom, R W. Taft, J. Mol. Struct. (Theochem) 1987, 153, 141-146.

[39] [39a] H. H. Jaffé, H. L. Jones, Adv. Heterocycl. Chem. 1964, 3 $209-261 .{ }^{[39 \mathrm{~b}]}$ P. Tomasik, C. D. Johnson, Adv. Heterocycl. Chem. 1976, 20,1-64.

${ }^{[40]}$ C. Eaborn, J. Organomet. Chem. 1975, 100, 43-57.

${ }_{141]}^{441 a]}$ J. Cretney, G. J. Wright, J. Organomet. Chem. 1971, 28, $49-52 .-{ }^{[416]}$ G. Seconi, M. Taddei, C. Eaborn, J. G. Stamper, J. Chem. Soc., Perkin Trans 2, 1982, 643-646.

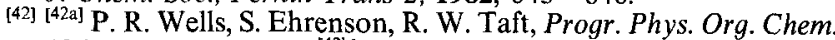
1968, 6, $147-322$. $-{ }^{[42 b]}$ S. Ehrenson, R. T. C. Brownlee, R. W Taft, Prog. Phys. Org. Chem. 1973, 10, 1-80. - ${ }^{[42 c]}$ C. Hansch, A. Leo, R. W. Taft, Chem. Rev. 1991, 91, 165-195.

${ }^{[41]}$ O. Exner in Correlation Analysis in Chemistry (Hrsg.: N. B. Chapman, J. Shorter), Plenum Press, New York, 1978, S. $439-540$.

${ }^{144]}{ }^{444 a]}$ J. A. Zoltewicz, R. E. Cross, J. Chem. Soc., Perkin Trans 2 , 1974, $1368-1373$. - ${ }^{[44 b]}$ I. F. Tupitsyn, N. N. Zatsepina, Y. M. Kapustin, A. V. Kirova, Reakts. Sposobnest Org. Soedin. 1968 , 5, 613-625, in Organic Reactivity 1968, 5, 248; Chem. Abstr. $1969,70,76941 \mathrm{y}$.

$[367 / 91]$ 